\title{
QoE-based Optimal Resource Allocation in Wireless Healthcare Networks: Opportunities and Challenges
}

\author{
Di Lin ${ }^{1}$, Fabrice Labeau ${ }^{2}$, Athanasios V.Vasilakos ${ }^{3}$ \\ ${ }^{1}$ School of Information and Software Engineering, University of Electronic Science and Technology of China \\ ${ }^{2}$ Department of Electrical and Computer Engineering, McGill University, Canada \\ ${ }^{3}$ Department of Computer and Telecommunications Engineering, University of Western Macedonia, Greece
}

\begin{abstract}
Ubiquitous health monitoring is a mobile health service with the aim of monitoring patients' conditions anytime and anywhere by collecting and transferring biosignal data from patients to health-service providers (e.g., healthcare centers). As a critical issue in ubiquitous health monitoring, wireless resource allocation can influence the performance of health monitoring, and the majority of work in wireless resource allocation for health monitoring has focused on quality-of-service (QoS) oriented allocation schemes with primary challenges at the physical and MAC layers. Recently, quality-of-experience (QoE) oriented resource allocation schemes in wireless health monitoring have attracted attentions as a promising design to a better service of healthcare monitoring. In this paper, we overview the metrics of assessing the quality of medical images, and discuss the performance of these metrics in QoE-oriented resource allocation for health monitoring. We start with addressing the state-of-theart QoE metrics by providing a taxonomy of the different metrics employed in assessing medical images. We then present the design of resource allocation schemes for health monitoring. After that, we present a case study to compare the performance of different classes of metrics in designing resource allocation schemes. We end the paper with a few open issues in the design of novel QoE metrics for resource allocation in health monitoring.
\end{abstract}

Index Terms-Wireless Health Monitoring, Resource Allocation, Quality of Experience

\section{INTRODUCTION}

Ubiquitous health monitoring is an emerging health service paradigm, which employs information and communications technologies to monitor patients' conditions anytime and anywhere with the aid of heterogeneous wireless networks [1]-[13]. In such an ubiquitous health monitoring system with heterogeneous networks (such as Wireless local area network (WLAN)/Wide area network (WAN)), the patients can access various wireless networks according to their availability, performance, and cost and can access the networks at any locations if only at least one type of wireless access is available there. The always-connected feature of ubiquitous health monitoring system can inform healthcare staff immediately when an emergency condition of patients occurs, and it can reduce the delay for medical treatment of a critical patient.

The quality of ubiquitous health monitoring service primarily depends on the quality of medical data at the receive end,

E-mail address: di.lin2@mail.mcgill.ca, fabrice.labeau@mcgill.ca, vasilako@ath.forthnet.gr. This work was partially supported by National Natural Science Foundation of China (No. 61370202) and partially supported by a grant from the National High Technology Research and Development Program of China (863 Program, No. 2012AA02A614) these data including both medical images, such as computed tomography (CT) and magnetic resonance imaging (MRI) images, and non-image data, such as blood pressure. While the quality of service (QoS) can assess the quality of nonimage data at the receiver, the criteria of assessing the quality of medical images needs to take into account the experience of people who will directly perceive, and therefore Quality of Experience (QoE) outperforms QoS when assessing the quality of medical images [14]-[21].

In order to guarantee the quality of medical data at the receive end, sufficient resources must be allocated to the transmission of a patient's medical data. However, with the increase of patients who try to access the wireless networks for healthcare monitoring, more consumption of wireless resources will lead to an increase of system cost, because the health-service provider must purchase the wireless connections from a telecommunications provider. Besides the concern of system cost, the spectrum shortage persists due to the increasing demand for more wireless bandwidth, and thus the limited amount of wireless resources (bandwidth, transmit power, ...) must be optimally allocated to support the health monitoring of patients within this network [22]-[29].

Most of the work in resource allocation for health monitoring has focused on the allocation of resources at lower layers of the protocol stack, mainly at the physical and MAC layers [30]-[32]. Their goal is to maximize the network capacity or minimize the system cost and meet the QoS/QoE requirements of data transmission. Also different from resource allocation in a regular communication system, resource allocation for health monitoring needs to consider the priority of patients, electromagnetic interference (EMI) on medical equipments, as well as unique QoS/QoE requirements for medical diagnosis purpose.

Assessing the quality of medical images is an important problem that influences the performance of QoE-oriented resource allocation for health monitoring. In the application of E-health, the primary goal of physicians and doctors reading a medical image is to reach a diagnosis conclusion. Thus, the metrics of assessing the quality of medical images are different from those of assessing the quality of a regular image, since a loss of one-pixel information in medical images might influence doctors' diagnosis if the lost information is critical, while the same issue might be trival to the visual perception in a regular image. In addition, most off-the-shelf metrics for assessing the quality of medical images center on diagnosis on 
one type of disease, and cannot be employed in the scenario whereby the same medical image might be reused to diagnose multiple diseases of a comorbid patient (e.g., a CT image for diagnosis on deep vein thrombosis and cataclasis). Such a need is especially clear for elderly patients: several studies have shown that about half of people 65 years or older have a comorbid condition [33]. In this paper, we present a survey and taxonomy of the different QoE metrics for QoE-oriented resource allocation in health monitoring and mention other notable aspects of such metrics. We highlight the challenges of designing a metric for assessing medical images, both inherited from assessing the quality of regular images and those unique to medical images for diagnosis purposes. We then provide a taxonomy of metrics in assessing the quality of medical images based on three main categories: objective metrics, subjective metrics, quasi-subjective metrics. Also we address the issues in the design of resource allocation schemes for healthcare monitoring. In view of these issues, we then compare the performance of various QoE metrics. Finally, we conclude the paper with a discussion of open issues in the design of new QoE metrics.

The rest of this paper is organized as follows: in Section II we address a taxonomy of different metrics employed in assessing the quality of medical images. Section III presents the factors that have to be addressed in designing resource allocation schemes for health monitoring. In Section IV we provide a case study that compares different classes of metrics in designing resource allocation schemes. Finally, we discuss future and open issues of designing novel QoE metrics which could be employed for resource allocation in health monitoring in Section V.

\section{TAXONOMY OF QOE METRICS}

The metrics of assessing the quality of medical images are different from those of assessing the quality of a regular image, since a loss of one pixel's information in medical images might influence a doctor's diagnosis if the lost information is critical, while the same issue might be trival to the visual perception in a regular image. And we refer to the metrics of assessing the quality of medical images for diagnosis on patient conditions as patient-diagnosis-oriented quality metrics (PDOQM).

From the perspective of whether involving visual perception of physicians for the assessment of image quality, off-the-shelf PDOQMs can be classified into three categories: (1) Objective metrics, (2) Subjective metrics, (3) Quasi-subjective metrics.

\section{A. Objective PDOQM}

Objective PDOQMs are usually characterized by a few numerical metrics which can well reflect the difference between the original and the received image.

Objective PDOQMs listed in Table I, especially MSE, PSNR, and SSIM are the most popular metrics used in medicalimage quality evaluation, especially in the real-time scenarios, since objective metrics can offer instant and consistent assessment results [34]-[39].

Arpah et al. in [34] employ the metrics of SNR and MSE to measure the quality of digital dental radiographs, in view

\begin{tabular}{|c|c|}
\hline List of indices & Description \\
\hline $\begin{array}{l}\text { Mean Square Error } \\
\text { (MSE) [34]-[36] }\end{array}$ & $\begin{array}{l}\text { Pixel difference between the } \\
\text { original and the received image }\end{array}$ \\
\hline $\begin{array}{l}\text { Peak Signal to Noise Ratio } \\
\text { (PSNR) [34], [37] }\end{array}$ & $\begin{array}{l}\text { Ratio of signal over the noise, } \\
\text { which is characterized by MSE }\end{array}$ \\
\hline $\begin{array}{l}\text { Structural Similarity Index } \\
\text { Metric (SSIM) [38] }\end{array}$ & $\begin{array}{l}\text { Measures the structural similarity } \\
\text { between two images }\end{array}$ \\
\hline Moran Peak Ratio (MPR) [39] & $\begin{array}{c}\text { A window based metric which measures } \\
\text { the spatial correlation }\end{array}$ \\
\hline Universal Quality Index [39] & Measures the structural distortion \\
\hline $\begin{array}{l}\text { Visual Information Fidelity } \\
\text { (VIF) [38], [39] }\end{array}$ & $\begin{array}{c}\text { Images are decomposed into wavelets to } \\
\text { measure the visual quality }\end{array}$ \\
\hline
\end{tabular}

TABLE I

OBJECTIVE QOE INDICES FOR ASSESSING MEDICAL-IMAGE QUALITY

of the differential diagnosis findings of intra-oral dental X-ray images for pre and post processing.

Istepanian et al. in [35] present a few objective metrics to assess the quality of images in a robotic teleultrasonography system applied in various clinical settings for remote ultrasound scanning without the need of the on-site physicians.

Vidhya et al. in [36] present the assessment the quality of medical images by objective metrics, and these objective metrics correlate well with the perceived image quality for the compression of DICOM (Digital Imaging and Communications in Medicine) images, which are for handling, storing, printing and transmitting information in medical imaging.

Ghrare et al. in [37] employ the metrics of MSE and PSNR to measure the quality of the reconstructed images in order to find out the answer of how much a typical medical image (CT, $\mathrm{X}$-ray, MRI) can be compressed and still preserve sufficient information for a clinical diagnosis.

Finn et al. in [38] present a detailed description and comparison of quality assessment methods for speckle reduction of medical ultrasound, and the preservation of image edges, overall image distortion, and improvement in image contrast are quantified by objective quality metrics.

Nakajima et al. in [39] present a few detailed evaluation methods of the motion picture compression, consisting of the frames with the different quality, such as I-picture, P-picture, and B-picture.

However, a shortcoming of such objective PDOQMs is that they sometimes cannot offer a reliable assessment of the quality of medical images for diagnosis, since these metrics do not take into account humans' visual perception in medical images. Thus, a high value of objective PDOQMs cannot ensure a superior quality of medical images for diagnosis [38].

\section{B. Subjective PDOQM}

Subjective PDOQMs are usually characterized by subjective assessments directly from physicians and doctors who provide their opinions on the quality of images for diagnosis. This assessment is done by referring to both the perceptual quality and the diagnostic information that can be extracted from the medical image.

Cosman et al. in [40] propose a subjective approach (MOS) to the measurement of medical image quality, and compare 


\begin{tabular}{l|c}
\hline List of indices & Description \\
\hline $\begin{array}{l}\text { Mean Opinion Score } \\
\text { (MOS) [40], [41] }\end{array}$ & $\begin{array}{c}\text { Given a randomized set of images, } \\
\text { experts rate these images in a scale of 1 to 5 }\end{array}$ \\
\hline Multiple Reader Multiple & $\begin{array}{c}\text { Medical experts read multiple cases } \\
\text { independently, and their opinion is collected }\end{array}$ \\
Case (MRMC) [42] & Medical expert are presented with \\
\hline Double Stimulus & two images side by side, typically \\
Continuous Quality & an original and received image \\
Scale (DSCQS) [43] &
\end{tabular}

TABLE II

SUBJECTIVE QOE INDICES FOR ASSESSING MEDICAL-IMAGE QUALITY

the subjective metric with the other objective measures in the particular application of compression in picture archiving and communication systems (PACS), determining diagnostic accuracy of lossy compressed medical images with various measures.

Pesce et al. in [41] present a survey of the recent use of receiver operating characteristic (ROC) analysis in medical imaging by reviewing ROC studies in Radiology for experimental design, imaging modality, medical condition, and ROC paradigm. ROC analysis is widely used in assessing diagnostic performance in Radiology, but not always adequate to support clear diagnosis conclusions.

Kim et al. in [42] propose a perceptual quality metric (defined as High Dynamic Range Visual Difference Predictor) to assess the quality of compressed abdominal transverse average-intensity-projection (AIP) images. The detailed process of estimating the image quality involves subjective scoring and friedman tests for paired proportions. By comparing the compressed and original images, three radiologists independently scored the images as 0 (indistinguishable), 1 (barely perceptible), 2 (subtle), or 3 (significant).

Duraisamy et al. in [43] presents the methods of contrast enhancement and evaluation of the Optical coherence tomography (OCT) images using various indices of image quality metrics, and also present the merits and demerits of subjective evaluation and objective measures.

Subjective PDOQMs listed in Table II, especially MOS, are the most popular metrics used in medical-image quality evaluation [40]-[43]. The advantage of subjective PDOQMs is that they can offer reliable assessment results, since these results are obtained based on the opinions of experts in medical field. However, a shortcoming of subjective PDOQMs for medical images is that these metrics sometimes cannot offer a consistent assessment of the quality of medical images, since different physicians and doctors may have different preferences in the assessment of image quality. Thus, the rate of assessment on the same image might be different in the second time by a different physician.

\section{Quasi-Subjective PDOQMs}

Subjective PDOQMs can offer reliable quality assessment of the medical images. However, their assessment results are often inconsistent and time-consuming. On the other hand, objective PDOQMs can reach to a consistent assessment conclusion immediatelty, however sometimes they are not reliable. Thus, there have been efforts made to develop novel PDOQMs (see Table III) which can take the advantages of both subjective and objective PDOQMs to further improve the assessment of medical image quality. This type of metrics is referred to Quasi-Subjective PDOQMs in this paper, because their process centers on how to approximate the assessment results by subjective PDOQMs in use of multiple objective metrics or in use of generalized objective metrics. Specifically, these types of metrics merge a few objective PDOQMs to approximate the subjective PDOQMs' assessment results for a high value of correlation between the assessment results by objective PDOQMs and by subjective PDOQMs.

Kumar et al. in [44] propose a method of finding the correlation between PSNR and Structural Similarity (SSIM) index objective image quality metrics with subjective MOS for SPIHT compressed medical images. The subjective assessment is based on the image quality scoring by six independent experts in medicine. It is found that correlation coefficient (CC) between the PSNR and MOS for CT scan images is higher than for MRI images, while the value of CC between the SSIM and MOS for CT scan images is lower than for MRI images.

Panayides et al. in [45] propose a quality assessment based on a clinical rating system which can offer the subjective evaluations of the different parts of clinical videos. They also estimate the correlation of objective video-quality assessment metrics to the subjective quality assessment. They point out the optimal objective quality assessment measures in each application scenario.

Przelaskowski et al. in [46] propose a vector measure of medical image quality, reflecting the accuracy of clinical diagnosis. This measure is construced by forming a diagnostic quality pattern extracted from the subjective scorings of ciritcal image features. The subjective rating process involves 9 radiologists: 2 test designers and 7 observers who graded digital mammograms. The correlation coefficient between the vector measure and the subjective pattern can reach up to 0.9.

Pambrun et al. in [47] study the quality assessment of compressed CT images. They present the diagnosis of compressed medical images and recommend the maximum acceptable compression ratios based on qualitative visual analysis. It is suggested that regular CT images cannot undergo lossy compression and still preserve sufficient information for diagnosis.

Kim et al. in [48] present the study of evaluating the compression ratio (CR), peak signal-to-noise ratio (PSNR), and a perceptual quality metric (high-dynamic range visual difference predictor, HDR-VDP) as indices of image fidelity for JPEG2000 compressed CT images. It is concluded that HDR-VDP is more suitable than the PSNR or CR as an measure of image quality for JPEG2000 compressed CT images.

All the aforementioned PDOQMs center on finding the optimal medical-image quality assessment metric for a diagnosis on one type of disease. However, these PDOQMs cannot be employed for the scenario in which the same medical image might be reused to diagnose multiple diseases of a comorbid patient (e.g., a CT image for diagnosis on deep vein thrombosis and cataclasis). Such a need is especially clear for 


\begin{tabular}{|c|c|c|}
\hline List of metrics & Description & $\begin{array}{c}\text { Correlation with } \\
\text { subjective metrics }\end{array}$ \\
\hline $\begin{array}{l}\text { B. Kumar, } \\
\text { et al. [44] }\end{array}$ & $\begin{array}{l}\text { Employed PSNR and } \\
\text { SSIM for SPIHT algorithm } \\
\text { and correlated with MOS }\end{array}$ & $\begin{array}{c}\text { PSNR and SSIM } \\
\text { showed } 0.86-0.98 \\
\text { correlation }\end{array}$ \\
\hline $\begin{array}{l}\text { A. Panayides, } \\
\text { et al. [45] }\end{array}$ & $\begin{array}{l}\text { SROCC and PLCC correlation } \\
\text { approaches were used to } \\
\text { correlate with MOS }\end{array}$ & $\begin{array}{l}\text { Best correlation } \\
\text { with PSNR } \\
\text { and WSNR }\end{array}$ \\
\hline $\begin{array}{l}\text { A. Przelaskowski } \\
\text { [46] }\end{array}$ & $\begin{array}{c}\text { Used MSE, PSNR, } \\
\text { Picture Quality Scale distortion } \\
\text { to correlate MOS }\end{array}$ & $\begin{array}{c}\text { The proposed } \\
\text { metrics showed a } \\
\text { correlation of } 0.9\end{array}$ \\
\hline $\begin{array}{l}\text { J. Pambrun, } \\
\text { et al. [47] }\end{array}$ & $\begin{array}{l}\text { Used VSNR to correlate } \\
\text { subjective scores for } \\
\text { JPEG } 2000\end{array}$ & $\begin{array}{l}\text { VSNR showed } \\
\text { a high correlation }\end{array}$ \\
\hline $\begin{array}{l}\text { K. J. Kim, } \\
\text { et al. }[48]\end{array}$ & $\begin{array}{c}\text { Used various objective metrics } \\
\text { with subjective metrics }\end{array}$ & $\begin{array}{l}\text { HDR-VDP metric } \\
\text { is best correlated }\end{array}$ \\
\hline $\begin{array}{l}\text { Di Lin, } \\
\text { et al. [49] }\end{array}$ & $\begin{array}{l}\text { Used for multiple } \\
\text { diagnosis purposes }\end{array}$ & $\begin{array}{c}\text { Reached to a } \\
\text { correlation of } 0.98\end{array}$ \\
\hline
\end{tabular}

TABLE III

QUASI-SUBJECTIVE PDOQMS FOR ASSESSING MEDICAL-IMAGE QUALITY

elderly patients: several studies have shown that about half of people 65 years or older have a comorbid condition [33]. Thus, in [49], an image quality metric (defined as metric for comorbidity, $I F C$ ) applicable for multiple diagnosis purposes is proposed, and it is able to assess how well a medical image can be used for the diagnosis on a comorbid patient's conditions as well as on a single disease of this patient. We summarize it here for completeness. IFC is designed by combining three factors of image distortion: luminance comparison, contrast comparison, and structure comparison between the original and compressed images.

Let $\mathbf{x}=\left\{x_{i} \mid i=1,2, \ldots, N\right\}$ and $\mathbf{y}=\left\{y_{i} \mid i=1,2, \ldots, N\right\}$ be the original and the compressed image signals, respectively. The proposed index $(I F C)$ is defined as

$$
e^{I F C}=k[L(\mathbf{x}, \mathbf{y})]^{a}[C(\mathbf{x}, \mathbf{y})]^{b}[S(\mathbf{x}, \mathbf{y})]^{c}
$$

where $L(\mathbf{x}, \mathbf{y})=2 \mu_{\mathbf{x}} \mu_{\mathbf{y}} /\left(\mu_{\mathbf{x}}^{2}+\mu_{\mathbf{y}}^{2}\right), C(\mathbf{x}, \mathbf{y})=2 \sigma_{\mathbf{x}} \sigma_{\mathbf{y}} /\left(\sigma_{\mathbf{x}}^{2}+\right.$ $\left.\sigma_{\mathbf{y}}^{2}\right), S(\mathbf{x}, \mathbf{y})=\sigma_{\mathbf{x y}} /\left(\sigma_{\mathbf{x}}+\sigma_{\mathbf{y}}\right)$ represent the luminance comparison, contrast comparison, and structure comparison, respectively. Also $\mu_{\mathbf{x}}$ and $\mu_{\mathbf{y}}$ represent the mean values of signals $\mathbf{x}$ and $\mathbf{y}$, and $\sigma_{\mathbf{x}}, \sigma_{\mathbf{y}}$, respectively; $\sigma_{\mathbf{x y}}$ represent the standard deviation of signal $\mathbf{x}$, the standard deviation of signal $\mathbf{y}$, and the convariance of signals $\mathbf{x}$ and $\mathbf{y}$, respectively.

Given luminance comparison, contrast comparison, and structure comparison, equation (1) is dependent on the values of constant $a, b, c$, and $k>0$. In the following, we are attempting to find the best values of $a, b, c$, and $k$ with the aid of curve fitting to ensure that the assessment result by the proposed index $(I F C)$ can best approximate the result by the subjective index of MOS (see Table II).

By tranforming the equation (1) with the logarithmic function of both sides, $I F C$ is shown as

$$
I F C=t+\operatorname{aln}[L(\mathbf{x}, \mathbf{y})]+b \ln [C(\mathbf{x}, \mathbf{y})]+\operatorname{cln}[S(\mathbf{x}, \mathbf{y})]
$$

where $t=\ln (k)$. Thus, we can employ linear curve fitting algorithms to find the optimal values of $a, b, c$, and $t$ to ensure that the assessment result can best approximate the MOS on medical images for patients in a comorbid condition.

\section{PRimitives OF RESOURCE ALLOCATION FOR HEALTH MONITORING}

\section{A. Electromagnetic interference in medical equipments}

The earliest research on EMI in hospital environments mainly focuses on the immunity of medical equipments to mobile phones. Tan et al. in [50] firstly propose that some types of medical equipments, such as ventilators, infusion pumps, and ECG monitors, are quite sensitive to the EMI from cellular phones. Then, an EMI susceptibility test was carried out by the Medicines and Healthcare Products Regulatory Agency (MHRA) of U.K. [51]; this test included testing the EMI of mobile phones and personal communication networks. The test results showed that external pacemakers, anesthesia machines, respirators, defibrillators are also susceptible to EMI. Trigano et al. in [52] and Calcagnini et al. in [53] study the EMI of GSM mobile phones on pacemakers and infusion pumps, respectively. Their results show that infusion pumps and pacemakers are inhibited due to the EMI of GSM mobile phones. With the implementation of $3 \mathrm{G}$ mobile phone systems in the United States, Japan, Hong Kong etc., the research of EMI effects on medical equipments in the $3 \mathrm{G}$ band has appeared [54], [55]. In 2007, the International Electrotechnical Committee (IEC) published the EN60601-12 standard, and the immunity levels are recommended as $3 \mathrm{~V} / \mathrm{m}$ and $10 \mathrm{~V} / \mathrm{m}$ for life-supporting equipments (e.g., blood pressure monitors and infusion pumps) and non-life-supporting equipments (e.g., defibrillators), respectively. In view of the advances of electromagnetic compatibility (EMC) technologies, some hospitals in Singapore and the U.K. relax the EMI restriction recommended in the EN60601-1-2 standard, and mobile phones are permitted to use in some areas of hospitals [56]. Chi-Kit et al. in [57] discuss the EMI test in view of the recently developed EMC of medical equipments, and the test takes into account the EMI of GSM900, PCS1800, and $3 \mathrm{G}$ mobile communication systems. The testing results show that ECG monitors, radiographic systems, audio evoked potential systems, and ultrasonic fetal heart detectors are sensitive to EMI [57]. Based on the previous literature, it can be concluded that the medical equipments sensitive to cellular phones include fetal monitors, infusion pumps, syringe pumps, ECG monitors, external pacemakers, respirators, anesthesia machines, and defibrillators [58].

The relevant literature focuses on the EMI on medical equipments from mobile phones, and the EMI effects from patient computing devices would be different. Wireless healthcare monitoring systems employ a wireless local area network, which usually works at the frequency band around $2.4 \mathrm{GHz}$. This frequency band is different from the frequency band which mobile phones work at, and the amount of EMI on a medical equipment is related to frequency bands. Given these reasons, the research on EMI in the scenario of wireless healthcare monitoring starts. Krishnamoorthy et al. in [59] measure the EMI on medical equipments from patient and doctor devices, which work around the $2.4 \mathrm{GHZ}$ frequency bands; the measurement is undertaken in two hospitals. The results show that the maximal EMI record is $0.552 \mathrm{~V} / \mathrm{m}$, which is within the acceptable EMI range recommended by the 
EN60601-1-2 standard. However, the measurement in [59] has not considered the QoS of data transmitted by patient devices and healthcare staff devices. The policy on mobile phone utilization, such as turning off mobile phone, cannot be applicable for patient devices and healthcare staff devices in a wireless healthcare monitoring system [60]. In wireless healthcare monitoring systems, healthcare staff and patients should employ wireless devices for data transmission and communication, and the restriction on transmit power may reduce the quality of service (QoS) of data transmission, which may increase the risk of medical data loss. Therefore, a contradiction between transmit power restriction and QoS requirements exists in wireless healthcare monitoring systems. In addition, when multiple patient devices and healthcare staff devices transmit data simultaneously, the aggregated signals at medical equipments would cause a higher level of EMI to medical equipments, including life-supporting equipments (e.g., blood pressure monitors and infusion pumps) and nonlife-supporting equipments (e.g., defibrillators) [61]. Phond et al. in [61] discuss the EMI in hospital environments, in view of the QoS of patient devices and healthcare staff devices. The conclusion is that EMI on most medical equipments is within the unacceptable range if the transmit power of a WLAN device is larger than $10 \mathrm{~mW}$.

For resource allocation, the restriction on EMI should be transformed into the constraint on transmit power, because transmit power, instead of EMI, is a type of resource which we would allocate. To the best of our knowledge, Phond et al. in [61] firstly propose the formula to calculate the maximal potential transmit power of a patient device subject to the EMI restriction. Medical equipments include life-support equipments and non-life-support equipments; different types of equipments may correspond to different requirements for the transmit power of a patient device and a healthcare staff device. The maximal potential transmit power should satisfy all these requirements (detailed in equation (3), equation (4), and equation (5)).

Patient devices and healthcare staff devices would cause interference to other devices and medical equipments. Medical equipments never cause interference to other equipments and devices. The interference between different equipments and devices is shown in Fig.1. In Fig.1, $A \rightarrow B$ means that $A$ would have interference on $B$. The interference on a lifesupport medical equipment and on a non-life-support medical equipment are represented by I and II, respectively. In addition, Phond et al. in [61] remark that the patient devices and healthcare staff devices may work within the same frequency bands. Therefore, the interference from a healthcare staff device on a patient device is also taken into account.

For a patient device, the potential interference or noise may be from the healthcare staff devices, the other patient devices, and the background noise of this patient device. The summation of all the potential interference or noise should be less than the tolerable level of interference. Mathematically,

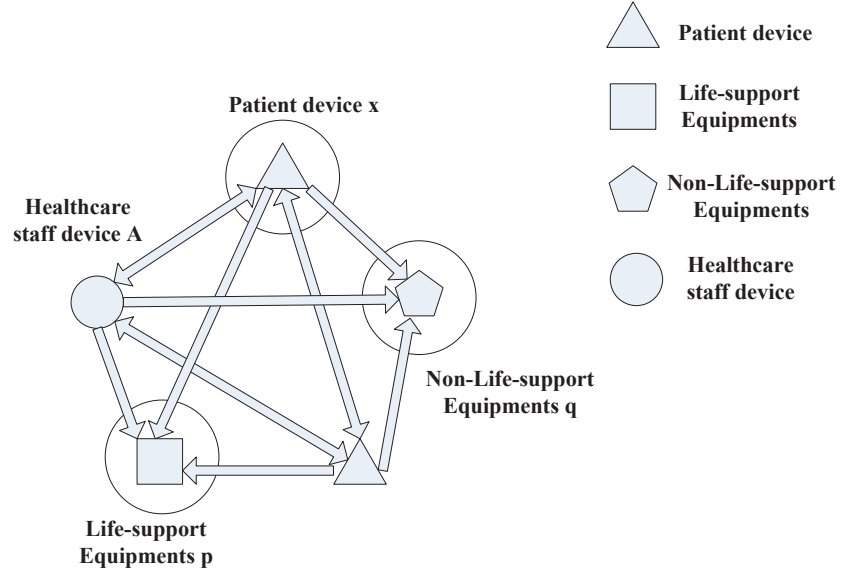

Fig. 1. Interference between different medical equipments and devices

for a patient device $x$, we have

$\frac{P_{t}(x)}{L\left(D_{x}(x)\right) \gamma(x)} \geq \sum_{A=1}^{A_{t}} \frac{P_{A}(x)}{L\left(D_{A}(x)\right)}+\sum_{z=1, z \neq x}^{X_{t}} \frac{P_{t}(z)}{L\left(D_{z}(x)\right)}+N(x)$

where $L(d)$ is the total indoor propagation path loss when the distance is $d ; P_{t}(x)$ is the transmit power of the patient device $x ; D_{x}(x)$ is the distance between the transmitter and the receiver of the device $x ; \gamma(x)$ is the SINR threshold of the device $x ; P_{A}(x)$ is the transmit power of a healthcare staff device $A ; D_{A}(x)$ is the distance between the healthcare staff device $A$ and the receiver of the patient device $x ; P_{t}(z)$ is the transmit power of a patient device $z(z \neq x) ; D_{z}(x)$ is the distance between the transmitter of the device $z$ and the receiver of the patient device $x ; N(x)$ is the background noise of the device $x ; X_{t}$ and $A_{t}$ are the number of patient devices and healthcare staff devices being turned on.

To analyze the cases of EMI on medical equipments, we should employ a basic relationship between radiated power $P[W]$ and electric field $E[V / m]$, that is, $E=Z \sqrt{P} / D$. $Z[\Omega]$ is the impedance of free space; $D[m]$ is the distance between the transmit and receive ends. The relationship between radiated power $P[W]$ and electric field $E[V / m]$ is recommended by IEC as $E=7 \sqrt{P} / D$ and $E=23 \sqrt{P} / D$ for a non-life-support equipment and for a life-support equipment, respectively. For a medical equipment, the summation of all potential interference should be less than the tolerable level of interference.

Mathematically, the constraints on transmit power can be shown in equation (4) and equation (5), for the cases of lifesupport medical equipments and of non-life-support medical equipments, respectively.

$$
\begin{aligned}
& \sum_{A=1}^{A_{t}} \frac{7 \sqrt{P_{N L S}(A)}}{D_{N L S}(A, p)}+\sum_{x=1}^{X_{t}} \frac{7 \sqrt{P_{t}(x)}}{D_{x}(p)} \leq E_{N L S}(p) \\
& \sum_{A=1}^{A_{t}} \frac{23 \sqrt{P_{L S}(A)}}{D_{L S}(A, q)}+\sum_{x=1}^{X_{t}} \frac{23 \sqrt{P_{t}(x)}}{D_{x}(q)} \leq E_{L S}(q)
\end{aligned}
$$


where $P_{N L S}(A)$ and $P_{L S}(A)$ are the maximal potential transmit power of a healthcare staff device $A$ to satisfy the EMI requirement of a non-life-support equipment and of a lifesupport equipment, respectively; $D_{N L S}(A, p)$ and $D_{L S}(A, q)$ are the distances from the healthcare staff device $A$ to the nonlife-support equipment $p$ and to the life-supporting equipment $q$, respectively; $E_{N L S}(p)$ and $E_{L S}(q)$ are the acceptable EMI levels for a non-life-support equipment $p$ and for a life-support equipment $q$, respectively; $P_{t}(x)$ is the transmit power of a patient device $x ; D_{x}(p)$ represents the distance between the transmitter of the device $x$ and the non-life-support $p ; D_{x}(q)$ represents the distance between the transmitter of the device $x$ and the life-support equipment $q ; X_{t}$ and $A_{t}$ are the number of patient devices and healthcare staff devices being working.

In a real hospital environment, patient devices, life-support medical equipments, and non-life-support medical equipments may operate at the same time. Therefore, the maximal potential transmit power of a healthcare staff device or a patient device should satisfy equation (3), equation (4), and equation (5).

\section{B. Channel characteristics in hospital environments}

Generally, the characteristics of a wireless channel are mainly determined by the communication environment as well as the communication technology. In our case, the communication environment refers to the hospital and mainly depends on the hospital's building materials. The building materials in a hospital usually have unique characteristics, such as their electromagnetic interference resistance, weather resistance, fire proofing, temperature adaptability, and environment friendliness [62]. Due to the unique characteristics of medical environment, the channel models widely used in general environments [63]-[65] are not applicable in medical environment. Additionally, even in the hospital environment, channel characteristics may also be different when a communication is at different wireless bands, which correspond to different attenuation of communication signals. We focus on using the IEEE 802.11n technology for communication within a hospital, and this technology employs the wireless bands around $2.4 \mathrm{GHz}$. Thus, the channel models at other wireless bands, such as those employed by the Oulu university hospital for ultra wideband applications at bands from $3.1 \mathrm{GHz}$ to $10.6 \mathrm{GHz}$ [66], are not applicable in our case.

To investigate the unique channel characteristics around 2.4GHz within a hospital, Huang and Francisco in [21] study the channel models in three LOS and one NLOS cases by taking channel measurements in the Kempenhaeghe Hospital, Heeze, the Netherlands. These three LOS cases include the transmission across the room (AR), the transmission along the front board of the bed $(\mathrm{AB})$, and the transmission along the bedside (AS) [67], while the NLOS case refers to the transmission through the bed. By matching the measurements and widely used channel models, Huang and Francisco in [67] conclude that the channel fading in all LOS and NLOS cases can be modeled as Nakagami distributions with particular parameters, and these parameters for various cases are shown in Table 2.1. The Nakagami distribution of channel fading $A$ can be expressed as

\begin{tabular}{|c|c|c|c|}
\hline \multirow{2}{*}{} & \multicolumn{3}{|c|}{ Distance(m) } \\
\cline { 2 - 4 } & 0.5 & 1 & 2 \\
\hline LOS/AR & 2.8998 & 1.684 & 1.6116 \\
\hline LOS/AS & 2.1665 & 1.6637 & 1.6356 \\
\hline LOS/AB & 1 & 1 & 1 \\
\hline NLOS & 1 & 1 & 1 \\
\hline
\end{tabular}

TABLE IV

PARAMETERS OF NAKAGAMI CHANNELS $(m)$ IN VARIOUS SCENARIOS FOR IN-HOSPITAL MONITORING [67]

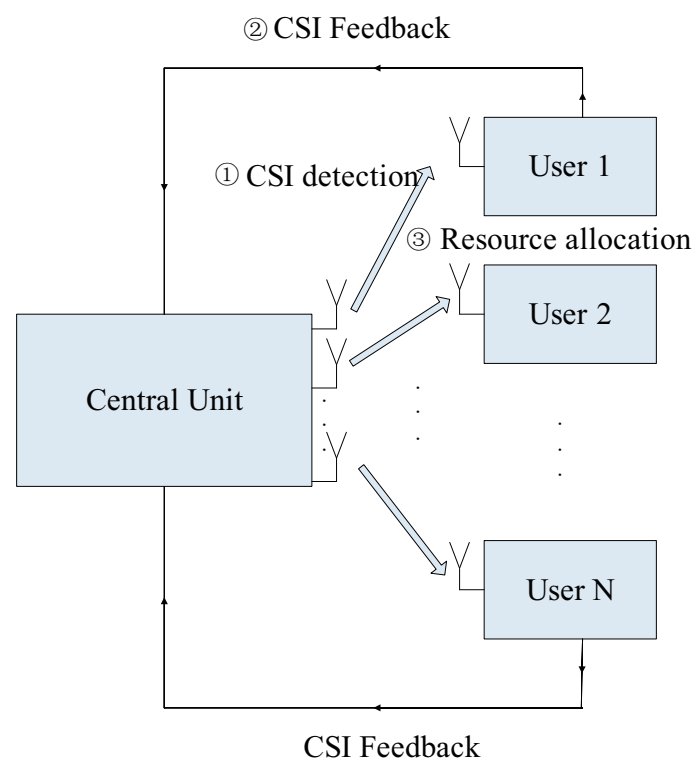

Fig. 2. Information flow of a centralized network [68] (C)2008 IEEE, reproduced with permission).

$$
f(A)=\frac{2 m^{m} A^{2 m-1}}{\sigma^{m} \Gamma(m)} e^{-m A^{2} / \sigma}
$$

where $\Gamma($.$) is a Gamma function; \sigma$ and $m$ are two determinant parameters of a Nakagami distribution.

\section{Imperfect channel state information}

The information flow for resource allocation in a centralized network is illustrated in Fig.2 [68]. Fig.2 shows that three steps are required for resource allocation in one time slot. The first step is the detection of various channels. Specifically, the central unit sends pilot signals, of which the amplitudes are known by all the users. Then, each user estimates the channel fading by comparing the amplitudes of received signals and the amplitudes of transmitted signals. The second step is the feedback of channel state information (CSI), that is, each user sends the estimated CSI to the central unit for resource allocation. In the third step, the central unit allocates resources among users based on the CSI feedback and, then, sends the decision of resource allocation to each user.

Based on the information flow shown in Fig.2, there are mainly five types of imperfect CSI. We will discuss these types of imperfect CSI and their respective causes.

The first type of imperfect CSI is caused by the errors of forward channel detection in step one, shown in Fig.2 [69], 
and we call it forward channel detection based imperfect CSI (ForCD-ICSI). In a noisy forward channel, a difference exists between the detected channel fading and the virtual channel fading. Therefore, the central unit cannot send an exact CSI to users.

The second type of imperfect CSI is caused by the errors of CSI feedback in step two, shown in Fig.2 [70], and we call it feedback based imperfect CSI (Fe-ICSI). In a noisy feedback channel, a difference exists between the detected channel fading and the virtual channel fading. Therefore, the central unit cannot receive the exact CSI feedback from a few users. In addition, if we employ the automatic repeat request (ARQ) in the upper layers, the channel detection errors also lead to a feedback delay. If the feedback delay is larger than a time slot for resource allocation, the central unit cannot obtain the detected CSI in the current time slot, which will lead to an inefficiency of resource allocation.

The third type of imperfect CSI is caused by the compression of feedback CSI at the user end [68], and we call it feedback compression based imperfect CSI (FC-ICSI). Due to the limitation of feedback bandwidth or the requirement of feedback delay, users usually employ as few feedback bits as possible to represent the feedback CSI; specific schemes include quantization and lossy compression [71]. Usually, the detected CSI will be quantized and compressed at the user end before sending to the central unit for a smaller amount of feedback CSI. Therefore, a loss of CSI in the received signals occurs at the central unit.

The fourth type of imperfect CSI is caused by the fast variation of channels and the feedback delay [71]-[73], and we call it fast-fading channel based imperfect CSI (FF-ICSI). Due to the doppler effect caused by the mobility of objects, channel fading would rapidly change. Because of the feedback delay, the estimated channel states may be different from the virtual CSI at the time of transmission. Therefore, even though the channel CSI in other parts of this system is perfect, the estimated CSI cannot represent the actual CSI at the time of transmission.

These four types of imperfect CSI summarized above are for the general centralized networks. As for the settings of applications in healthcare monitoring, only the first three types of imperfect CSI exist. The reasons are as follows: the FFICSI depends on the doppler effects, which is caused by the mobility of transmitters and receivers. However, in health monitoring settings, the speed of patients is quite low, and the doppler effect can be ignored [67]. Therefore, the fourth type of imperfect CSI, FF-ICSI, can be ignored in our analysis.

\section{DESIGNING QOE-ORIENTED RESOURCE ALLOCATION SCHEMES FOR HEALTH MONITORING}

\section{A. Network Model}

Ubiquitous mobile health monitoring systems are primarily designed for the monitoring of elderly patients who usually require long-term and intensive watching. This system is designed to deliver patients-related services and information via telecommunications and computing technologies. Once emergency conditions occur, healthcare staff must be alerted

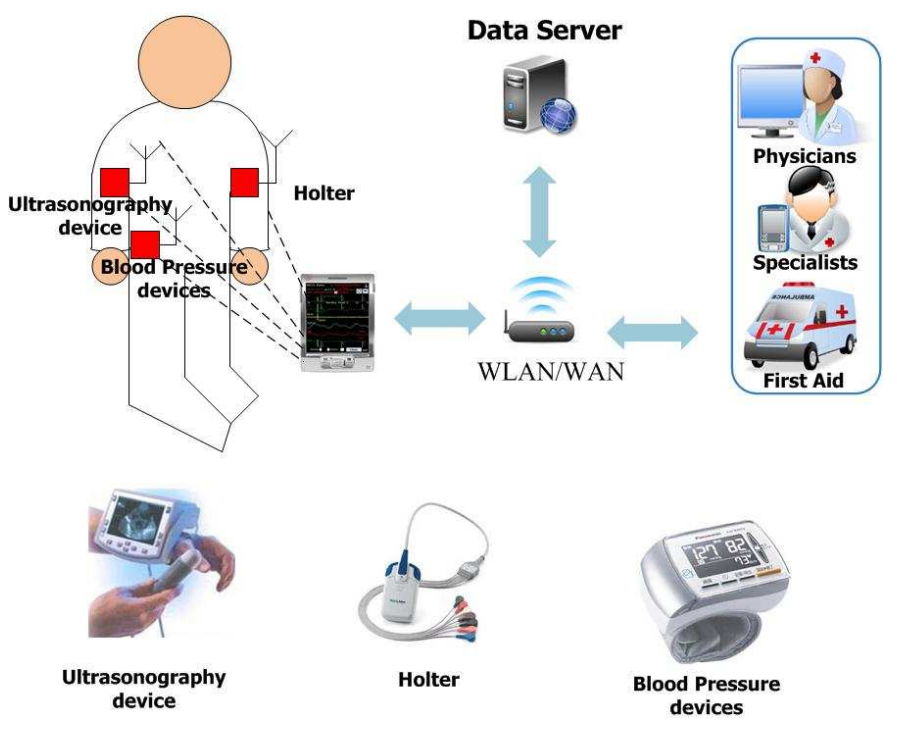

Fig. 3. Architecture of Health Monitoring Network

in time since a delay of even a few seconds may sometimes mean a loss of life.

To clarify the architecture of a health monitoring system (shown in Fig.3), we take the monitoring of patients with heart diseases as an example. At the beginning of monitoring, a patient would be required to wear a Holter device at his waist, shoulder or neck. The Holter device would collect the Electrocardiogram (ECG) of this patient and regularly send the ECG to a patient attached device, which might be a smart phone or a personal digital assistant (PDA). Then this patient attached device forwards the data to doctors for diagnosis as well as to a data server for filing. Also in the diagnosis of certain types of heart diseases, such as coronary artery diseases, doctor might skip the preliminaries and go straight to multislice computed tomography (CT) angiography, which will also be sent within the network for diagnosis and filing.

Thus, from the perspective of data transmission within the network, both medical images (e.g. CT images) as well as medical data (e.g. ECG data) might be sent to the end of physicians and specialists. For medical data, building on the status of patients, the data from different patient devices may have different QoS requirements and should be given different priorities. For medical images, QoS sometimes cannot reflect the humans' visual perception in medical images, so QoE, which takes into account the subjective perception, can better be used to assess the quality of medical images. In the application of health monitoring, the metrics of assessing the quality of medical images are different from those of assessing the quality of a regular image, since the former is designed for medical diagnosis. The detailed metric of assessing the quality of experience of medical images will be presented in Section III.D.

\section{B. Model on Patient Mobility Scenarios}

In a WAN/WLAN heterogeneous wireless network, the use of WLAN is always for free, while the use of WAN usually 


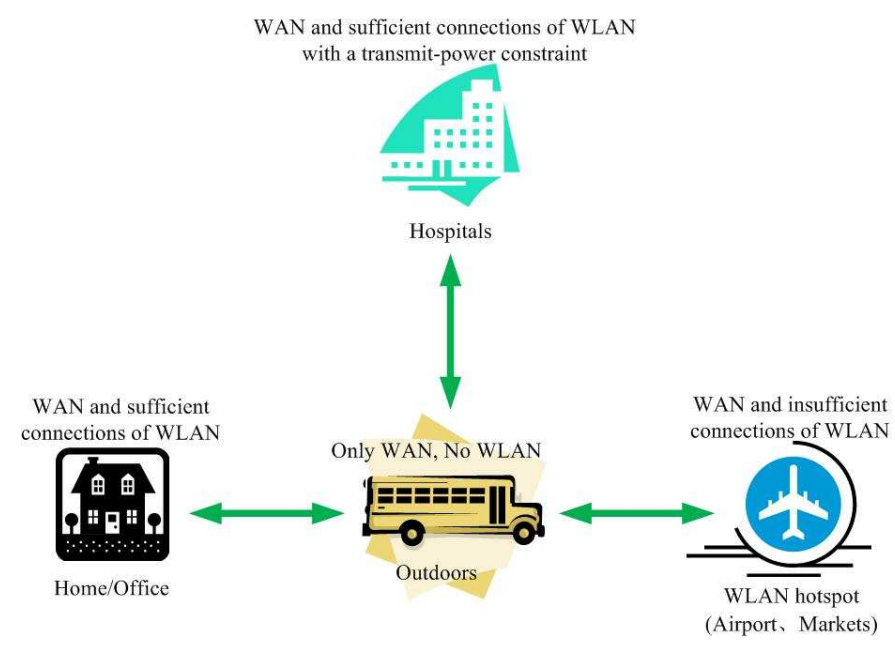

Fig. 4. Architecture of modelling patient mobility scenarios

leads to a network cost, because WAN connections must be purchased from telecommunications-service operators. In the following, from the perspective of reducing system cost, we assume that patients will first choose WLAN to access if available, and then choose WAN to access when no sufficient WLAN resources are available. The scenarios of health monitoring, shown in Fig.4, can mainly be classified into four categories: (1) Both WLAN and WAN are available and the resources of WLAN are sufficient (Scenario 1, S1), and this kind of scenario includes home or offices. (2) Both WLAN and WAN are available, but the resources of WLAN are insufficient due to many patients trying to access WLAN (Scenario 2, S2), this kind of scenario including WLAN hotspot, such as airport or market. (3) Both WLAN and WAN are available, but the transmit power of devices must be limited below a threshold (Scenario 3, S3), this kind of scenario including hospital, in which the transmit power cannot lead to a high level of Electromagnetic Interference (EMI) on medical equipments. (4) Only WAN is available and no WLAN is available (Scenario 4, S4), and this scenario includes ourdoors environment, such as in a park or on a vehicle.

\section{Transmission Scheduling at Patient-Attached Monitoring Device}

Without loss of generality, we assume that the priority of patients is descendingly ranked from 1 to $N_{u}$, that is, patient 1 has the highest priority, while patient $N_{u}$ has the lowest priority. Also we denote $B_{i}^{W L A N}$ and $B_{i}^{W A N}$ as the amount of wireless bandwidth used by patient $i$ to access WLAN and WAN, respectively. And we denote $B_{t o t}^{W L A N}$ and $B_{t o t}^{W A N}$ as the total amount of available wireless bandwidth for WLAN access and WAN access, respectively. For patients in scenario $S_{j}(j=1,2,3,4)$, we need to consider the following cases:

- Case 1: Both WLAN and WAN access are available (scenarios S1, S2, S3), such as home, hospital, hotspot scenarios in Fig.4. In this case, we need to first check whether WLAN bandwidth can support the data transmission of a particular patient as well as the patients with a higher priority. If so, the data of this patient will be sent via WLAN, which is the first choice if only it is available. Otherwise, we need to check whether this patient can send his or her data via WAN. Specifically, Case 1 is composed of three sub-cases:

- Case 1.1: if the total amount of bandwidth consumed by the first $i$ patients (those with highest priority) is smaller than the available amount of WLAN bandwidth, namely, $\sum_{j=1}^{i} B_{i}^{W L A N} \leq B_{\text {tot }}^{W L A N}$, then all the data of patient $i$ will be sent by WLAN.

- Case 1.2: if the total amount of WLAN bandwidth consumed by the first $i$ patients (those with highest priority) is larger than the available amount of WLAN bandwidth while the available amount of WLAN and WAN bandwidth can cover the need of bandwidth for the data transmission of first patient $i$, namely, $\sum_{j=1}^{i} B_{j}^{W L A N}>B_{\text {tot }}^{W L A N}$ and $\sum_{j=1}^{i} B_{j}^{W L A N}+\sum_{j=1}^{i} B_{j}^{W L A N} \leq B_{\text {tot }}^{W L A N}+B_{\text {tot }}^{W A N}$, then the amount of $\sum_{j=1}^{i} B_{j}^{W L A N}-B_{t o t}^{W L A N}$ WAN bandwidth will be consumed for the transmission of patient $i$ 's data, while the amount of $B_{i}^{W A N}-\left(\sum_{j=1}^{i-1} B_{j}^{W L A N}-B_{t o t}^{W L A N}\right)$ WLAN bandwidth will be consumed for the transmission of patient $i$ 's data.

- Case 1.3: if the total amount of bandwidth consumed by the first $i$ patients (those with highest priority) is larger than the available amount of WAN and WLAN bandwidth, namely, $\sum_{j=1}^{i} B_{j}^{W L A N}+\sum_{j=1}^{i} B_{j}^{W L A N}>B_{\text {tot }}^{W L A N}+B_{\text {tot }}^{W A N}$, then patient $i$ cannot transmit the data until a few patients who have a higher priority finish their data transmission.

- Case 2: Only WAN access is available (scenario S4), such as the outdoors scenarios in Fig.4. In this case, we need to check whether WAN bandwidth can support the data transmission of a particular patient as well as the patients with a higher priority. Specifically, Case 2 is composed of two subcases:

- Case 2.1: if the total amount of bandwidth consumed by the first $i$ patients (those with highest priority) is smaller than the available amount of WAN bandwidth, namely, $\sum_{j=1}^{i} B_{j}^{W A N} \leq B_{\text {tot }}^{W A N}$, then all the data of patient $i$ will be sent by WAN.

- Case 2.2: if the total amount of bandwidth consumed by the first $i$ patients (those with highest priority) is larger than the available amount of WAN bandwidth, namely, $\sum_{j=1}^{i} B_{j}^{W A N}>B_{\text {tot }}^{W A N}$, then patient $i$ cannot transmit the data until a few patients who have a higher priority finish their data transmission.

\section{Optimization of Heterogeneous Wireless Resource}

If the number of patients within a health-service area exceeds the network capacity of WLAN, then WAN connections will need to be deployed within the area. While WLAN 


\section{Patients in Health}

Service Area

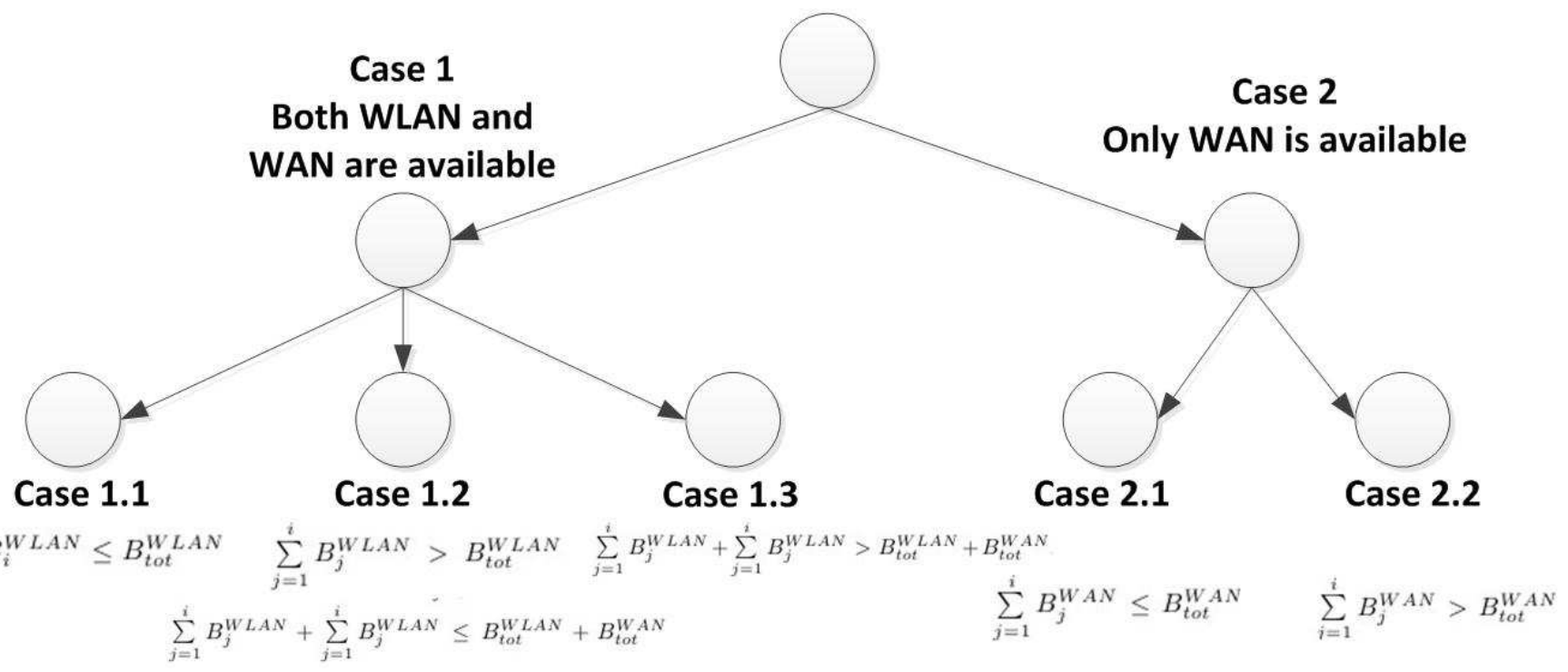

Fig. 5. The different cases for connections from patients

connections are usually free for use, WAN connections must be purchased from telecommunications-service operators. Beyond the cost of deploying WAN networks, their co-existence might also reduce their individual performance in a few healthservice locations (e.g., Scenario S3 in Fig.4) in which a potential impact of EMI on medical equipments from both WAN and WLAN networks may force to reduce the transmit power of patient attached device to access either WLAN or WAN. These considerations lead us, in this paper, to attempt to minimize the amount of WAN deployment, considering real-world health-service scenarios as well as QoS and QoE requirements.

Let $B_{i}^{W L A N}[H z]$ and $B_{i}^{W A N}[H z]$ represent the amount of bandwidth consumed by patient $i$ when accessing WLAN and WAN, respectively; $B_{\text {tot }}^{W L A N}[H z]$ and $B_{t o t}^{W A N}[H z]$ represent the total amount of available bandwidth for patient $i$ when accessing WLAN and WAN, respectively; $a_{i}[b p s]$ is the transmission rate of patient $i$ 's data; $T_{c}[s]$ is the length of a data-transmission slot; $\eta_{i}^{W L A N}[b p s / H z]$ and $\eta_{i}^{W A N}[b p s / H z]$ represent the bandwidth efficiency for the transmission of patient $i$ 's data when accessing WLAN and WAN, respectively; $\Delta T_{i}^{W L A N}[s]$ and $\Delta T_{i}^{W A N}[s]$ represent the length of patient $i$ accessing WLAN and WAN, respectively; $\Delta T_{i}^{W L A N}[s]$ represents the tolerable delay of data transmission for patient $i$; $P_{i}^{W L A N}[W]$ and $P_{i}^{W A N}[W]$ represent the transmit power of patient device attached to patient $i$ when accessing WLAN and WAN, respectively; $P_{\max }^{W L A N}(i)[W]$ and $P_{\max }^{W A N}(i)[W]$ represent the maximal potential transmit power of patient device attached to patient $i$ when accessing WLAN and WAN, respectively; $S c(i)$ is a binary variable, a value of 1 when patient $i$ in scenario S3 and a value of 0 when patient $i$ in the other scenarios; $\operatorname{Im}(i)$ is a binary variable, a value of 1 when patient $i$ transmitting medical images and a value of $\infty$ when patient $i$ transmitting the other types of data; $M E T_{i}^{W L A N}$ and $M E T_{i}^{W A N}$ represent the value of metrics to assess the quality of medical images of patient $i$ when accessing WLAN and WAN, respectively; $M E T_{a c c}(i)$ represents the acceptable value of metrics for the diagnosis on patient $i$ 's condition; $r_{i}^{W L A N}$ and $r_{i}^{W A N}$ represent the signal to noise ratio (SNR) for patient $i$ when accessing WLAN and WAN, respectively; $h_{i}^{W L A N}$ and $h_{i}^{W A N}$ represent the channel fading when transmitting the data of patient $i$ via WLAN and WAN, respectively; $\sigma^{2}[W / H z]$ is the noise spectral density; $a_{k, l}^{\prime}$ is a coefficient determined by curve fitting; $E_{l}(x)=\int_{1}^{\infty} e^{-x t} t^{-l} d t$, and it is defined as the $l$-order exponential integral function of $x$.

Building on the aforementioned notations, the problem of minimizing the amount of WAN bandwidth can be modeled as a programming problem. Given the distribution of patient scenarios $\pi$ (see Section IV A), this problem can be mathematically denoted as $l(\pi)$ in (7): 


$$
\begin{aligned}
& l(\pi): \min _{B_{i}^{W A N}} \sum_{i=1}^{N_{u}} B_{i}^{W A N} \\
& \text { s.t. } \sum_{i} B_{i}^{W L A N} \leq B_{\text {tot }}^{W L A N} \\
& \sum_{i=1}^{N_{u}} B_{i}^{W A N} \leq B_{t o t}^{W A N} \\
& a_{i} T_{c} \leq \eta_{i}^{W L A N} B_{i}^{W L A N} \Delta T_{i}^{W L A N}+\eta_{i}^{W A N} B_{i}^{W A N} \Delta T_{i}^{W A N} \\
& \Delta T_{i}^{W L A N}+\Delta T_{i}^{W A N} \leq \Delta T_{i} \\
& M E T_{i}^{W L A N} I m(i) \geq M E T_{a c c}(i) \\
& M E T_{i}^{W A N} \operatorname{Im}(i) \geq M E T_{a c c}(i) \\
& P_{i}^{W L A N} S c(i) \leq P_{m a x}^{W L A N}(i) \\
& P_{i}^{W A N} S c(i) \leq P_{\max }^{W A N}(i) \\
& r_{i}^{W L A N}=P_{i}^{W L A N}\left|h_{i}^{W L A N}\right|^{2} /\left(B_{i}^{W L A N} \sigma^{2}\right) \\
& r_{i}^{W A N}=P_{i}^{W A N}\left|h_{i}^{W A N}\right|^{2} /\left(B_{i}^{W A N} \sigma^{2}\right) \\
& \eta_{i}^{W L A N}=\left[\sum_{p=1}^{m} \exp \left\{p / r_{i}^{W L A N}\right\} \sum_{l=n-m}^{(n+m-2 p) p} a_{p, l}\right. \\
& \left.\quad \times \sum_{j=1}^{l+1} E_{l+2-j}\left(p / r_{i}^{W L A N}\right)\right]_{i} \\
& \eta_{i}^{W L A N}=\left[\sum_{p=1}^{m} \exp \left\{p / r_{i}^{W A N}\right\} \sum_{l=n-m}^{(n+m-2 p) p} a_{p, l}\right. \\
& \left.\quad \times \sum_{j=1}^{l+1} E_{l+2-j}\left(p / r_{i}^{W A N}\right)\right]_{i} \\
& B_{i}^{W L A N} / \Delta B \in Z^{+}, B_{i}^{W A N} / \Delta B \in Z^{+}
\end{aligned}
$$

The objective (3a) is to find the minimal amount of WAN bandwidth consumed by all the patients, namely, $\sum_{i=1}^{N_{u}} B_{i}^{W A N}$. Constraints (7b) and (7c) ensure that the total amount of bandwidth consumed by patients does not exceed the total amount of available bandwidth when accessing WLAN and WAN, respectively. Constraints (7d) and (7e) impose delay requirements for patient data, either medical images or non-image data. Constraints (7f) and (7g) impose imagequality requirements when patients are transmitting medical images for doctors' diagnosis. Constraints (7h) and (7i) show the limit of transmit power when accessing WLAN and WAN, respectively. Constraints (7j), (7k), (71), (7m) characterize the signal-to-noise ratio as well as bandwidth efficiency as functions of transmit power ${ }^{1}$. Constraint (3n) ensures that the bandwidth consumed must be a non-negative integer multiple of the bandwidth of a connection $\Delta B$, since in the real world, telecommunications operators always sell wireless connections instead of wireless bandwidth.

\section{Comparative Study}

In this section, we compare three of the listed classes of QoE metrics in the previous section under various scenarios via Matlab simulations. The main purpose of this section is to present how we choose a metric and under which scenario each metric would be suitable.

\footnotetext{
${ }^{1}$ Equations (7l) and (7m) hold when the wireless network is with a MIMOOFDM architecture, and we assume that both WLAN and WAN networks for health service employ this architecture.
}

\begin{tabular}{l|c|c|c}
\hline Compression methods & MOS1 & MOS2 & MOS \\
\hline SPIHT-3D & 3.2 & 3.0 & 3.0 \\
\hline SPIHT & 4.4 & 4.6 & 4.4 \\
\hline SOTW & 4.6 & 4.8 & 4.6 \\
\hline LVL-MMC & 4.0 & 3.8 & 3.8 \\
\hline GBL-MMC-H & 1.6 & 1.4 & 1.4 \\
\hline
\end{tabular}

TABLE V

MOS OF MEDICAL IMAGES WITH VARIOUS COMPRESSION METHODS.

\begin{tabular}{l|c|c|c|c}
\hline Correlation & MSE & PSNR & SSIM & IFC \\
\hline MOS & 0.9127 & 0.8775 & 0.9690 & $\mathbf{0 . 9 9 8 7}$ \\
\hline MOS1 & 0.9283 & 0.8523 & 0.9570 & $\mathbf{0 . 9 9 8 5}$ \\
\hline MOS2 & 0.8962 & 0.8971 & 0.9766 & $\mathbf{0 . 9 9 9 0}$ \\
\hline Running time [s] & MSE & PSNR & SSIM & IFC \\
\hline MOS & $\mathbf{0 . 2 3}$ & 0.34 & 1.98 & 2.21 \\
\hline MOS1 & $\mathbf{0 . 2 1}$ & 0.32 & 1.95 & 2.18 \\
\hline MOS2 & $\mathbf{0 . 2 5}$ & 0.37 & 1.99 & 2.23 \\
\hline
\end{tabular}

TABLE VI

CORRELATION \& RUNNING TIME BETWEEN RESULTS BY OBJECTIVE METRICS AND BY MOS.

\section{A. Scenarios of simulation}

We consider the following scenario: a patient who suffers from deep vein thrombosis also experiences Cataclasis, and diagnosis on both of them needs a CT image. The original CT image is compressed with the methods of SPIHT-3D, SPIHT, SOTW, LVL-MMC, GBL-MMC-H when arriving at the receive end. As a benchmark for comparing various metrics of objective or Quasi-objective QoE, we invite five family doctors in a local hospital to rate the quality of 30 medical images using 1-5 scale as bad, poor, fair, good and excellent, and then calculate their MOS, which is shown in Table V. MOS represents rating the quality of images for the diagnosis on both deep vein thrombosis and Cataclasis, MOS1 represents rating the quality of images for the diagnosis on deep vein thrombosis, MOS2 represents rating the quality of images for the diagnosis on Cataclasis.

\section{B. Comparison of QoE metrics}

Building on the results of MOS, we compare the correlation between the metric by various metrics and the value of MOS to determine which metric can best approximate subjective rating, and the comparison results are shown in Table VI. As shown in Table VI, the value of correlation between IFC and MOS is higher than its peers, and thus IFC outperforms than the other objective metrics to approximate the MOS metric for medical diagnosis. This metric is appropriate to be used as a criterion to measure the QoE of medical-image transmission and can serve as a constraint in the programming problem of minimizing system cost (see constraints (7f) and $(7 g)$ ).

However, the running time of IFC is longer than the other metrics when assessing the quality of images, while MSE takes the shortest running time (see Table VI). Thus, MSE is more applicable for real-time scenarios in which the running time of assessing images is a critical factor. 
C. Performance of resource allocation with various QoE metrics

We consider a health-service area in Fig. 2 with 20 patients and each patient has an identical mobility model, which is characterized as the following probability transition matrix $\mathbf{P}_{i}\left(i=1,2 \ldots, N_{u}\right)$ :

$\mathbf{P}_{i}=\left(\begin{array}{cccc}0.9998333 & 0 & 0 & 0.0001667 \\ 0 & 0.9996667 & 0 & 0.0003333 \\ 0 & 0 & 0.9999167 & 0.0000833 \\ 0.0003333 & 0.0001667 & 0.0001667 & 0.9993333\end{array}\right)$

In this scenario, the average durations for which a patient will be in scenario 1 , scenario 2, scenario 3, and scenario 4 are 200, 100, 25 and 50 minutes, respectively. The packet arrival processes of medical-image and non-image medical data are assumed to be Bernoulli with the probabilities of 0.1 and 0.8 , which represent the probability of image data and non-image data are generated in a transmission slot. The length of a transmission slot is 1 minute. Also we assume that the data (either image and non-image data) of patients can be classified as Emergency and Normal (corresponding to Emergency and Normal condition of patients, respectively). The delay requirements of the Emergency and Normal data are 2 and 5 transmission slots, respectively; And the lowest acceptable values of metrics for Emergency and Normal image data are 4.9 and 4.4 (1-5 scaled metric), respectively.

The consumption of WAN bandwidth based on QoE assessed by various metrics is shown in Fig.6. When QoE of medical images are assessed by IFC, the consumption of WAN is closest to the that based on QoE assessed by MOS. Thus, from the perspective of WAN consumption, IFC can best approximate MOS, and outperforms the other metrics of assessing the quality of medical images. The running time of resource allocation based on QoE by various metrics is listed in Fig.7. As shown in Fig.7, MSE and PSNR based resource allocation take the shortest running time. Thus, in practice, we need to balance the running time and the performance of resource allocation.

\section{Impact of QoE requirements}

In this section, we investigate how the change of QoS or QoE requirements impacts health-service cost, namely, the amount of WAN bandwidth consumption. In view that medical data include both medial images and non-images, we consider how the change of QoS and QoE requirements influences the consumption of WAN bandwidth, respectively.

Firstly, we investigate how the change of delay requirements impacts the amount of consumed WAN bandwidth, namely, how much WAN bandwidth has to be consumed in order to support the transmission delay at an acceptable level. Assuming that $\Delta T_{i}=\Delta T / 2$ if patient $i$ is in emergency condition, while $\Delta T_{i}=\Delta T$ if patient $i$ is in normal condition, then the change of WAN bandwidth consumption with $\Delta T$ is shown in Fig.8.

As shown in Fig.8, with the increase of required delay for data transmission, the ratio of WAN bandwidth consumption

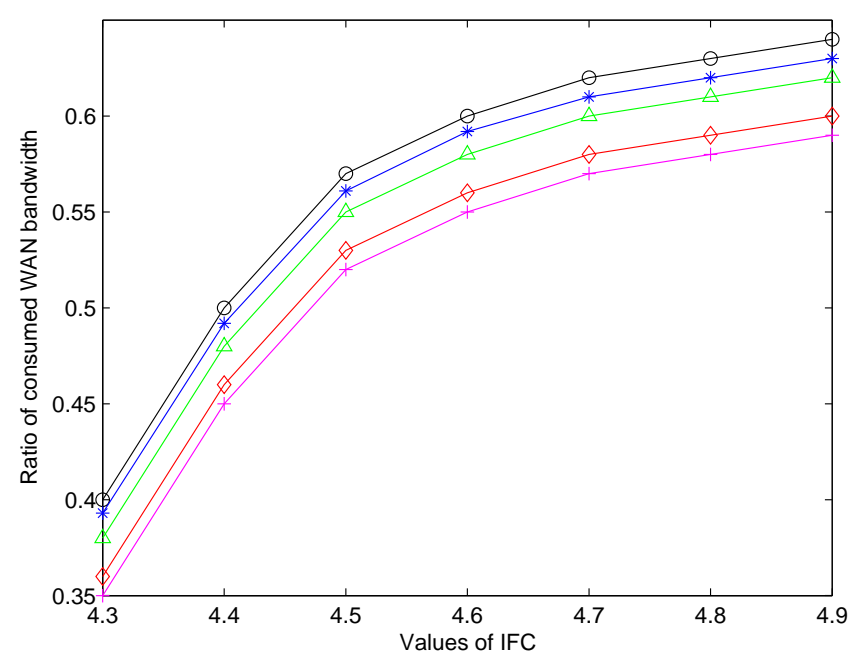

Fig. 6. Ratio of consumed WAN bandwidth (The dark line with o represents MOS; The blue line with $*$ represents IFC; The green line with $\triangle$ represents SSIM; The red line with $\diamond$ represents PSNR; The purple line with + represents MSE.)

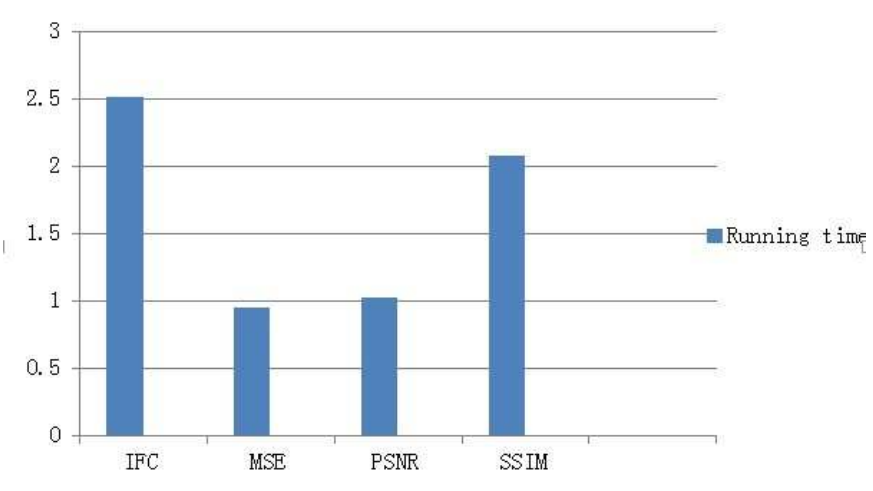

Fig. 7. Running time of resource allocation based on QoE by various metrics [s]

to the total amount of available WAN bandwidth decreases and converges to a certain level which depends on the value of $I F C$. When the value of delay increasing from $5 \mathrm{~s}$ to $35 \mathrm{~s}$, the ratio of WAN consumption varies around $12 \%$.

Then, we investigate how the amount of WAN bandwidth consumption varies with the values of $I F C$, which measures the acceptable quality of medical images at the receive end. As shown in Fig.9, the ratio of WAN bandwidth consumption to the total amount of available WAN bandwidth increases with the values of $I F C$ and also converges to a certain level depending on the value of $\Delta T$. When the value of $I F C$ increasing from 4.3 to 4.9 , the ratio of WAN consumption varies around $24 \%$.

In consideration of Fig.8 and Fig.9, both the values of $\triangle T$ and $I F C$ will influence the ratio of WAN bandwidth consumption, and the change of bandwidth consumption is more dependent on the value of $I F C$ than on the value of $\Delta T$. The aforementioned results on system cost can help the health- 


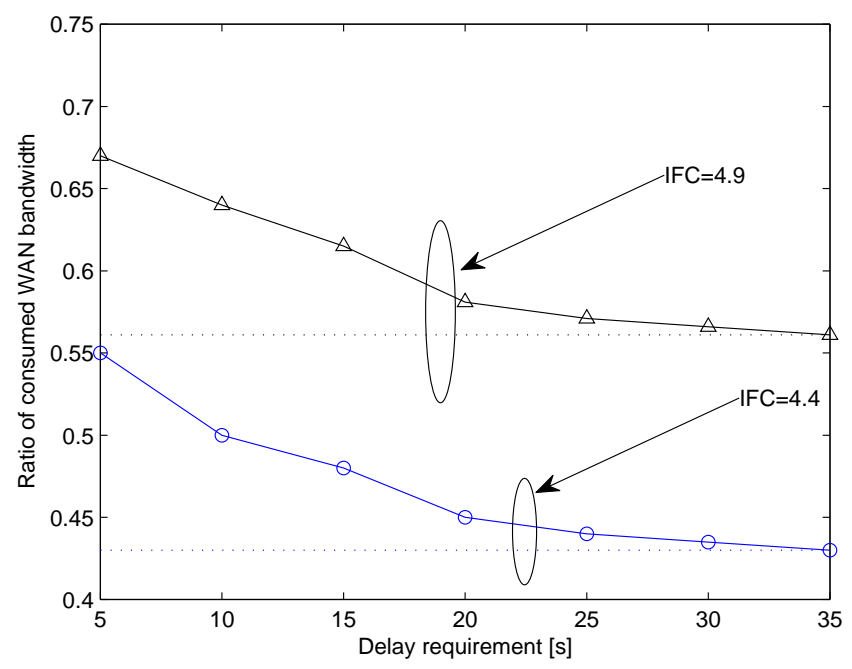

Fig. 8. Impact of transmission delay requirements on the consumption of WAN bandwidth (The blue line with o represents the case when $I F C=4.4$; The black line with $\triangle$ represents the case when $I F C=4.9$.)

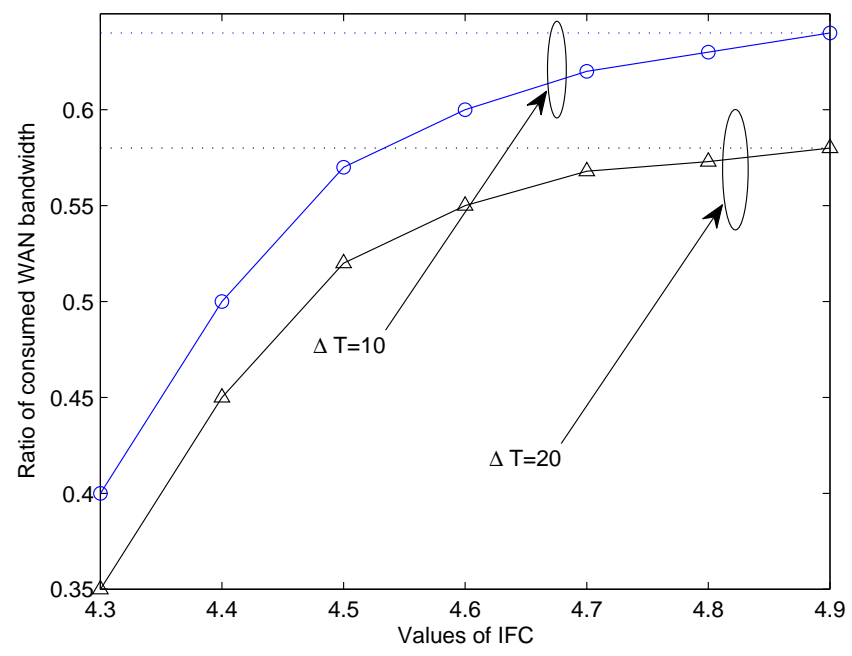

Fig. 9. Impact of IFC values on the consumption of WAN bandwidth (The blue line with o represents the case when $\Delta T=10 \mathrm{~s}$; The black line with $\triangle$ represents the case when $\Delta T=20 \mathrm{~s}$.)

service provider provide flexible and cost-effective monitoring service to mobile patients.

\section{E. Solution to the problem of resource allocation}

Given each patient moving among 4 kinds of scenarios in Fig. 4, the objective of problem (5) is a summation of $4 \times N_{u}$ items, subject to $4 \times N_{u} \times 13$ constraints. This problem with a small scale (a small value of $N_{u}$ ) can be solved by Branch and Bound approach, and the detailed algorithm of Branch and Bound is shown in Algorithm 1.

Although the aforementioned Branch and Bound algorithm offers an optimal solution, solving the programming problem with this algorithm is computationally expensive and thus
Input: The optimization problem with the objective of minimizing $\sum_{i=1}^{N_{u}} B_{i}^{W A N}$

Output: The result of this algorithm, $\bar{B}^{W A N}=\left[B_{1}^{W A N}, B_{2}^{W A N}, \cdots B_{N_{u}}^{W A N}\right]$

1 We create a relaxation of the problem (5) by omitting constraints $(4 \mathrm{j})$ and $(4 \mathrm{k})$. ;

2 Solve the relaxed problem and the solution is denoted as $\bar{B}_{1}^{W A N}, \bar{B}_{2}^{W A N}, \cdots \bar{B}_{N_{u}}^{W A N}$;

3 If any $\bar{B}_{(i)}^{W A N} / \Delta B$ is not an integer, then, we split the problem (5) into two sub-problems with the constraints $B_{i}^{W A N} \geq\left[\bar{B}_{i}^{W A N} / \Delta B\right] \Delta B$ and $B_{i}^{W A N} \leq \bar{B}_{i}^{W A N} / \Delta B \mid \Delta B$, respectively; Go to step 4. Otherwise, $\bar{B}^{W A N}=\left[\bar{B}_{1}^{W A N}, \bar{B}_{2}^{W A N}, \cdots \bar{B}_{N_{u}}^{W A N}\right]$ and output the solution. ;

4 Repeat the steps $1-3$ until $\bar{B}^{W A N}$ is found.

Algorithm 1: Branch and Bound algorithm for wireless resource allocation

done in an off-line manner, especially when the scale of this problem is large (a large value of $N_{u}$ ). However, if the scenarios of patients and requirements of their data transmission vary dynamically over time, an on-line algorithm would be needed. Therefore, we investigate an alternative approximation algorithm based on Genetic theory which is much simpler to implement. This approximation algorithm learns and adapts the scheduling action by calculating the system cost due to the price paid for WAN connections, shown in Algorithm 2. Also as shown in [74], the solution by Algorithm 2 can converge in mean to the optimal solution.

As shown in Algorithm 1 and 2, both Branch and Bound algorithm (off-the-shelf) as well as Genetic Theory based algorithm (Proposed) are based on multiple iterations, and the convergence of both to the optimum solution is shown in Fig.10. Fig.10 shows that the proposed scheduling approach can save the consumed WAN bandwidth, and in fact it can reduce the ratio of consumed WAN bandwidth (to the total available WAN bandwidth, i.e. $\sum_{i=1}^{N_{u}} B_{i}^{W A N} / B_{\text {tot }}^{W A N}$ ) from $70 \%$ to $45 \%$. Thus, the proposed scheduling approach can save the WAN bandwidth and thus reduce the health-service cost. Also Fig.10 shows that both Branch and Bound algorithm as well as the proposed algorithm can converge to the optimal solution, but the convergence rate of the proposed algorithm is higher than the Branch and Bound algorithm. Specifically, when the number of iterations reach to around 400, the proposed algorithm has almost converged to the optimal solution, while the peer number has to reach around 800 for Branch and Bound algorithm. Thus, the Genetic Theory based algorithm can greatly reduce the computation time and is appropriate for online computation scenarios.

\section{Future Open Issues}

In this section, we discuss open issues in the design and implementation of QoE-based resource allocation and future directions. 
Input: The optimization problem with the objective of minimizing $\sum_{i=1}^{N_{u}} B_{i}^{W A N}$

Output: The result of this algorithm,

$$
\bar{B}^{W A N}=\left[B_{1}^{W A N}, B_{2}^{W A N}, \cdots B_{N_{u}}^{W A N}\right]
$$

1 We randomly select $N_{0}$ of $\bar{B}^{W A N}$ from the set of $\bar{B}^{W A N}$ that satisfy all the constraints of equation (5); we denote them as $\bar{B}_{(1)}^{W A N}, \bar{B}_{(2)}^{W A N}, \cdots \bar{B}_{\left(N_{0}\right)}^{W A N}$;

2 Order all the $\bar{B}_{(j)}^{W A N}\left(j=1,2, \cdots N_{0}\right)$ for an ascending order of objective function $\sum_{i=1}^{N_{u}} B_{i}^{W A N}$, and we denote ordered $\bar{B}_{(j)}^{W A N}$ as $\overline{\bar{B}}_{(1)}^{W A N}, \overline{\bar{B}}_{(2)}^{W A N}, \ldots, \overline{\bar{B}}_{\left(N_{0}\right)}^{W A N}$;

3 Set the fitness function as $f_{j}=\operatorname{fitness}\left(\overline{\bar{B}}_{(j)}^{W A N}\right)=p_{f}\left(1-p_{f}\right)^{j-1}\left(j=1,2 \cdots N_{0}\right)$, where $0 \leq p_{f} \leq 1$;

4 Calculate the probability $p_{j}=f_{j} / \sum_{j=1}^{N_{0}} f_{j}$, and we reselect $N_{0}$ of $\bar{B}^{W A N}$ from the set of $\overline{\bar{B}}_{(1)}^{W A N}, \overline{\bar{B}}_{(2)}^{W A N}, \cdots, \overline{\bar{B}}_{\left(N_{0}\right)}^{W A N}$, with the probability of $p_{j}$ to select $\overline{\bar{B}}_{(j)}^{W A N}$. The same $\overline{\bar{B}}_{(j)}^{W A N}$ is allowed to be selected multiple times, and we denote the reselected group as $\underline{\bar{B}}_{(1)}^{W A N}, \underline{\bar{B}}_{(2)}^{W A N}, \cdots, \underline{B}_{\left(N_{0}\right)}^{W A N}$;

5 With a probability $p_{c}$ of inheritance, we generate the next generation of $\underline{\bar{B}}_{(j)}^{W A N}$ according to $\underline{\bar{B}}_{(i)}^{W A N}=C \underline{\bar{B}}_{\left(j_{1}\right)}^{W A N}+(1-C) \underline{\bar{B}}_{\left(j_{2}\right)}^{W A N}$, where $\underline{\bar{B}}_{(i)}^{W A N}$ denotes the next generation of $\underline{\bar{B}}_{(j)}^{W A N}, C$ is randomly selected between 0 and $1, \underline{\bar{B}}_{\left(j_{1}\right)}^{W A N}$ and $\underline{\bar{B}}_{\left(j_{2}\right)}^{W A N}$ are a pair of $\underline{\bar{B}}_{(j)}^{W A N}$ selected for inheritance. If $\underline{\bar{B}}_{(i)}^{W A N}$ satisfying all constraints of equation (5), then, we keep it in the next generation; otherwise, we reselect the pair of $\underline{B}_{(j)}^{W A N}$ for inheritance until we get $\underline{\bar{B}}_{(i)}^{W A N}\left(i=1,2, \cdots N_{0}\right)$; 6 With a small probability $p_{m}$, mutation of some $\underline{\bar{B}}_{(i)}^{W A N}$ would occur. The process of mutation is $\underline{\underline{B}}_{(i)}^{W A N}=\underline{\bar{B}}_{(i)}^{W A N}+M_{(i)}^{W A N} d_{(i)}^{W A N}$, where $M_{(i)}$ is a randomly selected step size and $d_{(i)}$ is a randomly selected direction of the $N_{0}$-dimension space formed by vectors $\underline{\bar{B}}_{(i)}^{W A N}$. If $\underline{\underline{B}}_{(i)}^{W A N}$ satisfying all the constraints of equation (5), then, we keep it in this generation; Otherwise, we would regenerate $M_{(i)}^{W A N}$ and $d_{(i)}^{W A N}$ until we get a $\underline{\underline{\bar{B}}}_{(i)}^{W A N}$ that satisfy all the constraints of equation (5);

7 Record $\overline{\underline{B}}_{(i)}^{W A N}$ that maximizes the objective function up to $k$ th generation (Also $k$ th time slot, because we assume one generation is generated within one time slot);

8 Repeat all the steps above, and output the $\underline{\underline{\bar{B}}}_{(i)}^{W A N}$.

Algorithm 2: Genetic theory based algorithm for wireless resource allocation

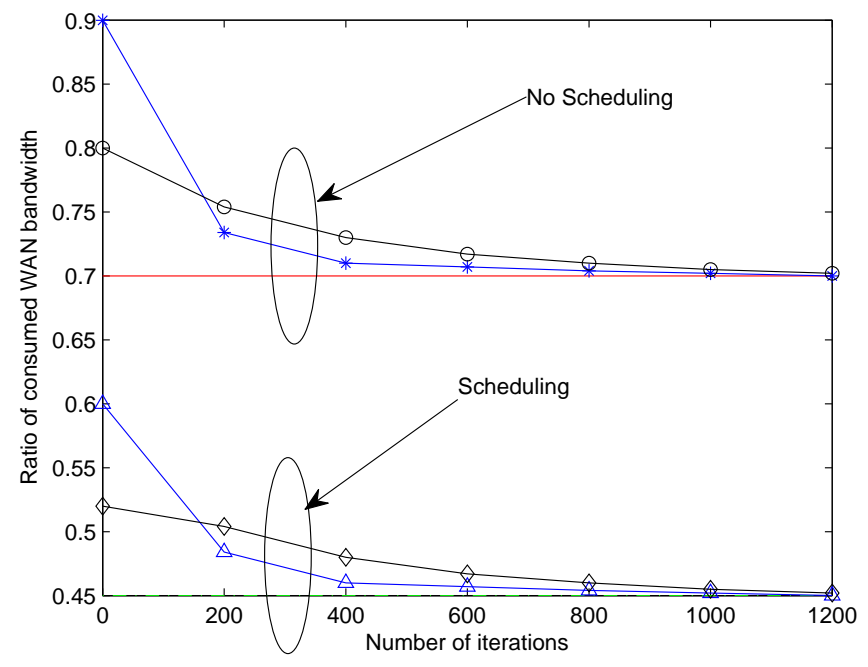

Fig. 10. Convergence of Genetic Theory based Algorithm (The blue line with $*$ represents Genetic Theory based Algorithm without scheduling; The blue line with $\triangle$ represents Genetic Theory based Algorithm with scheduling; The black line with o represents Branch and Bound Algorithm without scheduling; The black line with $\diamond$ represents Branch and Bound Algorithm with scheduling; The red line represents the optimal solution without scheduling; The green dotted line represents the optimal solution with scheduling.)

A. Metrics for assessing the quality of images for multiple diagnosis purposes

In the field of medicine, a medical image might be reused for multiple diagnosis purposes, for example, a CT image for diagnosis on deep vein thrombosis can also be used for the diagnosis on cataclasis. Such a need is especially clear for elderly patients: several studies have shown that about half of people 65 years or older have a comorbid condition. However, most off-the-shelf metrics for assessing the quality of medical images are designed for a diagnosis on one type of disease, and these different metrics might be applicable for assessing the quality of images for different diagnosis purposes. Thus, designing a general QoE metric with varying parameters can be used for multiple diagnosis purposes, whereby the metric with one optimal parameter can be used to assess the quality of images for the diagnosis of one disease. When applying the QoE metric into the process of resource allocation, we can allocate the resources to patients by referring to their types of diseases, and design an individualized QoE metric for each patient.

\section{B. Context-awareness metrics for assessing the quality of images}

When designing the metrics of assessing the quality of images, we also need to take into account the context of patients. The critical factor of QoE assessment in the scenario of large-scale emergency rescue, such as in an earthquake, could be different from the factor in the scenario of regular health monitoring. In the former case, to guarantee the quality of images of emergency patients, the quality of images of the other less emergent patients can be reduced. In other words, 
the level of acceptable quality of images must be adjusted dynamically with the context of a particular patient. The issue of designing dynamic metrics for assessing QoE is still an open issue and should be developed in the future research.

\section{Metrics for assessing the quality of videos for medical applications}

Video remote interpreting (VRI) is a recently growing field of application in medicine, especially in the hospital emergency room. In such a setting, patients and caregivers who cannot communicate readily in a common language need an interpreter, but it may take time for a live interpreter to arrive onsite. Hospitals with VRI capability can connect with a remote interpreter quickly and conduct triage and intake surveys with the patient or caregiver without significant delay. Assessing the quality of VRI for medical application in consideration of subjective rating is still an open issue, and the metric of VRI assessment should be developed [75], [76].

\section{CONCLUSION}

In this paper, we made an overview on the QoE-based resource allocation in wireless networks for health applications, and classify the metrics of $\mathrm{QoE}$ assessment into subjective metrics, objective metrics as well as quasi-subjective metrics. We discussed the architecture of resource allocation, including network modelling, patient flow modelling, as well as data tranmission scheduling. We reviewed and compared the existing metrics of QoE assessment and also pointed out the open research issues with the hope to spark new interests and advances in this area.

\section{ACKNOWLEDGE}

This work is supported by National Natural Science Funds of China (No. 61370202).

\section{REFERENCES}

[1] Robson B., Baek O. K. "The engines of Hippocrates: From the Dawn of Medicine to Medical and Pharmaceutical Informatics", John Wiley \& Sons, 2009.

[2] Rashvand, H. F., V. T. Salcedo. "Ubiquitous wireless telemedicine", IET Communications, 2(2): 237-254, 2008.

[3] Dowler N. and Hall C.J. "Safety issues in telesurgery -summary", IEE Colloquium on 'Towards Telesugery', 1995.

[4] Choi Y. B., J. S. Krause. "Telemedicine in the USA: standardization through information management and technical applications", IEEE Communications Magazine, 44(4): 41-48, 2006.

[5] Istepanian R. S., H. E. Jovanov. "Guest Editorial Introduction to the Special Section on M-Health: Beyond Seamless Mobility and Global Wireless Health-Care Connectivity", IEEE Transactions on Information Technology in Biomedicine, 8(4): 405-414, 2004.

[6] S.E.Kern, D. Jaron. "Healthcare technology, economics and policy: an evolving balance", IEEE Engineering in Medicine and Biology Magazine, 22(1): 16-19, 2003.

[7] Vasilakos A. V., C. Hsiao-Hwa. "Guest editorial wireless and pervasive communications for healthcare", IEEE Journal on Selected Areas in Communications, 27(4): 361-364, 2009.

[8] Golmie N., D. Cypher. "Performance analysis of low rate wireless technologies for medical applications", Computer Communications, 28(10): 1266-1275, 2005.

[9] Varshney U., S. Sneha. "Patient monitoring using ad hoc wireless networks: reliability and power management", IEEE Communications Magazine, 44(4): 49-55, 2006.
[10] Varshney U. "A framework for supporting emergency messages in wireless patient monitoring", Decision Support Systems, 45(4): 981-996, 2008.

[11] Sneha S., U. Varshney. "Enabling ubiquitous patient monitoring: Model, decision protocols, opportunities and challenges", Decision Support Systems, 46(3): 606-619, 2009.

[12] Vergados D. J., D. D. Vergados. "NGL03-6: Applying Wireless DiffServ for QoS Provisioning in Mobile Emergency Telemedicine", IEEE Global Telecommunications Conference, 2006.

[13] Cypher D., N. Chevrollier. "Prevailing over wires in healthcare environments: benefits and challenges", IEEE Communications Magazine, 44(4): 56-63, 2006.

[14] Giovanni Acampora, Diane J. Cook, Parisa Rashidi, Athanasios V. Vasilakos. "A Survey on Ambient Intelligence in Healthcare", Proceedings of the IEEE, vol 101, no 12, pp 2470-2494, 2013.

[15] Min Chen, Sergio Gonzalez, Athanasios Vasilakos, Huasong Cao, Victor C. M. Leung. "Body Area Networks: A Survey", MONET 16(2): 171193, 2011.

[16] Jun Zhou, Xiaodong Lin, Athanasios Vasilakos. "Securing m-healthcare social networks: challenges, countermeasures and future directions", IEEE Wireless Commun, 20(4), 2013.

[17] He D, Chen C, Chan S, Bu J, Vasilakos AV. "ReTrust: Attack-Resistant and Lightweight Trust Management for Medical Sensor Networks", IEEE Transactions on Information Technology in Biomedicine, 16(4): 623-632, 2012.

[18] He D, Chen C, Chan S, Bu J, Vasilakos AV. "A Distributed Trust Evaluation Model and Its Application Scenarios for Medical Sensor Networks", IEEE Transactions on Information Technology in Biomedicine, 16(6): 1164-1175, 2012.

[19] Zhaoyang Zhang, Honggang Wang, Athanasios V. Vasilakos, and Hua Fang. "ECG-Cryptography and Authentication in Body Area Networks", IEEE Transactions on Information Technology in Biomedicine 16(6): 1070-1078, 2012.

[20] Naixue Xiong, A. Vasilakos, L. Yang, Lingyang Song, Yi Pan, R. Kannan, Yingshu Li. "Comparative analysis of quality of service and memory usage for adaptive failure detectors in healthcare systems", IEEE Journal on Selected Areas in Communications 27(4): 495-509, 2009.

[21] Thaier Hayajneh, Ghada Almashaqbeh, Sana Ullah, Athanasios V. Vasilakos. "A survey of wireless technologies coexistence in WBAN: analysis and open research issues", ACM/Springer WINET, DOI 10.1007/s 11276-014-0736-8.

[22] G Acampora, et al: "A Survey on Ambient Intelligence in Healthcare". Proceedings of the IEEE, 101(12): 2470-2494, 2013.

[23] Jun Zhou, et al: "Securing m-healthcare social networks: challenges, countermeasures and future directions". IEEE Wireless Commun. 20(4), 2013.

[24] Daojing He, et al: ReTrust: Attack-Resistant and Lightweight Trust Management for Medical Sensor Networks. IEEE Transactions on Information Technology in Biomedicine 16(4): 623-632, 2012.

[25] Daojing He, et al: A Distributed Trust Evaluation Model and Its Application Scenarios for Medical Sensor Networks. IEEE Transactions on Information Technology in Biomedicine 16(6): 1164-1175, 2012.

[26] Naixue Xiong, et al: Comparative analysis of quality of service and memory usage for adaptive failure detectors in healthcare systems. IEEE Journal on Selected Areas in Communications 27(4): 495-509, 2009.

[27] Giancarlo Fortino, Giuseppe Di Fatta, Mukaddim Pathan, Athanasios V. Vasilakos: Cloud-assisted body area networks: state-of-the-art and future challenges. Wireless Networks 20(7): 1925-1938, 2014.

[28] Thaier Hayajneh, Ghada A. Al-Mashaqbeh, Sana Ullah, Athanasios V. Vasilakos: A survey of wireless technologies coexistence in WBAN: analysis and open research issues. Wireless Networks 20(8): 2165-2199, 2014.

[29] Zhaoyang Zhang et al: ECG-Cryptography and Authentication in Body Area Networks. IEEE Transactions on Information Technology in Biomedicine 16(6): 1070-1078, 2012.

[30] Phond Phunchongharn, E. H., Sergio Camorlinga. "Electromagnetic Interference-Aware Transmission Scheduling and Power Control for Dynamic Wireless Access in Hospital Environments, IEEE Transactions on Information Technology in Biomedicine, 15 (6): 890-899, 2011.

[31] Phunchongharn P., Niyato D., Hossain E., Camorlinga S. "Robust Transmission Scheduling and Power Control for Dynamic Wireless Access in a Hospital Environment, IEEE International Conference on Communications (ICC): 1-5, 2011.

[32] Phunchongharn P., Niyato D., Hossain E., Camorlinga S. "Robust Transmission Scheduling and Power Control for Dynamic Wireless 
Access in a Hospital Environment", IEEE International Conference on Communications (ICC): 1-5, 2001.

[33] Wilk, Sz., Michalowski, W., Michalowski, M., Farion, K. and MainegraHing, M. "Mitigation of Adverse Interactions in Pairs of Clinical Practice Guidelines using Constraint Logic Programming", Journal of Biomedical Informatics, 46(2): 341-353, 2013.

[34] S. Arpah, B. Ahmad, M. N. Taib, N. E. A. Khalid, and H. Taib. "Analysis of image quality based on dentists' perception cognitive analysis and statistical measurements of intra-oral dental radiograph", in International Conference on Biomedical Engineering, pp. 379- 384, 2012.

[35] R. S. H. Istepanian, N. Philip, M. G. Martini, N. Amso, and P. Shorvon. "Subjective and objective quality assessment in wireless teleultrasonography imaging", in 30th Annual International Conference of the IEEE Engineering in Medicine and Biology Society, 2008.

[36] K. Vidhya and S. Shenbagadevi. "Performance Analysis of Medical Image Compression", in International Conference on Signal Processing Systems, 2009.

[37] S. E. Ghrare, M. A. M. Ali, M. Ismail, and K. Jumari. "Diagnostic Quality of Compressed Medical Images: Objective and Subjective Evaluation", in Second Asia International Conference on Modeling and Simulation, 2008.

[38] S. Finn, M. Glavin, and E. Jones. "Echocardiographic speckle reduction comparison", IEEE Transactions on Ultrasonics, Ferroelectrics and Frequency Control, vol. 58, no. 1, pp. 82-101, 2011.

[39] I. Nakajima, K. Nakada, Y. Tomioka, H. Juzoji, and T. Kitano. "Methods for performing video quality evaluations in the field of medicine", in IEEE Region 10 Conference TENCON, 2010.

[40] P. C. Cosman, R. M. Gray, and R. A. Olshen. "Evaluating Quality of Compressed Medical Images: SNR, Subjective Rating and Diagnostic Accuracy", Proceedings of the IEEE, vol. 82, no. 6, pp. 919-932, 1994.

[41] L. L. Pesce, C. E. Metz, and K. Doi. "Experimental design and data analysis in receiver operating characteristic studies: Lessons learned from reports in Radiology from 1997 to 2006", Radiology, vol. 253, no. 3, pp. 822-830, 2009.

[42] B. Kim, K. H. Lee, K. J. Kim, R. Mantiuk, H. R. Kim, and Y. H. Kim. "Artifacts in slab average-intensity-projection images reformatted from JPEG 2000 compressed thin-section abdominal CT Data sets", American Journal of Roentgenology, vol. 190, no. 6, pp. 342-350, 2008.

[43] P. Duraisamy, X. Yuan, A. E. Saba, and S. Palanisamy. "Contrast enhancement and assessment of OCT images", in International Conference on Informatics, Electronics and Vision, pp. 91-95, 2012.

[44] B. Kumar, S. P. Singh, A. Mohan, and H. V. Singh. "MOS Prediction of SPIHT Medical Images Using Objective Quality Parameters", in International Conference on Signal Processing Systems, 2009.

[45] A. Panayides, M. S. Pattichis, C. S. Pattichis, C. C. Loizou, M. Pantziaris, and A. Pitsillides. "Atherosclerotic Plaque Ultrasound Video Encoding, Wireless Transmission, and Quality Assessment Using H.264", in IEEE Transactions on Information Technology in Biomedicine, vol. 15, no. 3, pp. 387-397, 2009.

[46] A. Przelaskowski. "Vector quality measure of lossy compressed medical images" Computers in biology and medicine, vol. 34, no. 3, pp. 193-207, 2004.

[47] J. F. Pambrun and R. Noumeir. "Perceptual quantitative quality assessment of JPEG2000 compressed ct images with various slice thicknesses", in IEEE International Conference on Multimedia and Expo (ICME), 2011.

[48] K. J. Kim, K. H. Lee, H. S. Kang, S. Y. Kim, Y. H. Kim, B. Kim, J.Seo, and R. Mantuik. "Objective metric of image fidelity for JPEG2000 compressed body CT Images", Medical Physics, vol. 36, no. 7, 2009.

[49] Di Lin, Fabrice Labeau, Xuanli Wu. "Quality metric of Medical Images for multiple diagnosis purposes", Submitted.

[50] K.-S. Tan and I. Hinberg. "Radiofrequency susceptibility tests on medical equipment", IEEE 16th Annu. Int. Conf, 2: 998-999, 1994.

[51] Electromagnetic compatibility of medical devices with mobile communications. in Medical Devices Bulletin DB9702. London, U.K.: Medical Devices Agency, 1997.

[52] A. J. Trigano, A. Azoulay,M. Rochdi, and A. Campillo. "Electromagnetic interference of external pacemakers by walkie-talkies and digital cellular phones: experimental study", Pacing Clin. Electrophysiol, 22(4): 588-593, 1999.

[53] G. Calcagnini, P. Bartolini, M. Floris, M. Triventi, P. Cianfanelli, G. Scavino, L. Proietti, and V. Barbaro. "Electromagnetic interference to infusion pumps from GSM mobile phones", IEEE 26th Annu. Int. Conf, 2: 3515-3518, 2004.

[54] Y. Chu and A. Ganz."A mobile teletrauma system using $3 \mathrm{G}$ networks", IEEE Trans. Inf. Technol. Biomed, 8(4): 456-462, 2004.
[55] E. A. V. Navarro, J. R.Mas, J. F. Navajas, and C. P. Alcega. "Performance of a 3G-based mobile telemedicine system", IEEE 3rd Consum. Commun. Netw. Conf, 2: 1023-1027, 2006.

[56] E-Health Insider. DH to lift hospital mobile phone ban. http://www.ehealth-insider.com/news/item.cfm? ID=2542, 2007.

[57] Chi-Kit T., C. Kwok-Hung. "Electromagnetic Interference Immunity Testing of Medical Equipment to Second- and Third-Generation Mobile Phones", IEEE Transactions on Electromagnetic Compatibility, 51(3): 659-664, 2009.

[58] Ardavan M., K. Schmitt. "A preliminary assessment of EMI control policies in hospitals", 14th International Symposium on Antenna Technology and Applied Electromagnetics and the American Electromagnetics Conference, 2010.

[59] Krishnamoorthy S., J. H. Reed. "Characterization of the $2.4 \mathrm{GHz}$ ISM band electromagnetic interference in a hospital environment", Proceedings of the 25th IEEE Annual International: 3245-3248, 2003.

[60] Witters D., S. Seidman. "EMC and wireless healthcare", Asia-Pacific Symposium on Electromagnetic Compatibility, 2010.

[61] Phond Phunchongharn, D. N., Ekram Hossain and Sergio Camorlinga. "An EMI-Aware Prioritized Wireless Access Scheme for e-Health Applications in Hospital Environments", IEEE transactions on information technology in biomedicine, 14(5): 1247-1258, 2010.

[62] Hanada E, Watanabe Y, Antoku Y, Kenjo Y, Nutahara H, Nose Y. "Hospital construction materials: poor shielding capacity with respect to signals transmitted by mobile telephones", Biomed Instrum Technol, 32(5): 489-496, 1998.

[63] Walker E., H. J. Zepernick. "Fading measurements at $2.4 \mathrm{GHz}$ for the indoor radio propagation channel", International Zurich Seminar on Broadband Communications, 1998.

[64] Zepernick H. J., T. A. Wysocki. "Multipath channel parameters for the indoor radio at $2.4 \mathrm{GHz}$ ISM band", IEEE Vehicular Technology Conference, 1999.

[65] Robert Akl, D. T., Xinrong Li. "Indoor propagation modeling at 2.4 GHZ for IEEE 802.11 networks", The sixth international multi-conference on wireless and optical communications networks and emerging technologies, 2006.

[66] L. Hentila, A. Taparungssanagorn, H. Viittala, M. Hamalainen. "Measurement and modelling of an UWB channel at hospital", IEEE Intl. Conf. Ultra-WideBand (ICUWB), 2005.

[67] L. Huang, R. d. F., and G. Dolmans. "Channel measurement and modeling in medical environments", Third International Symposium on Medical Information and Communication Technology, 2009.

[68] Love D. J., R. W. Heath. "An overview of limited feedback in wireless communication systems", IEEE Journal on Selected Areas in Communications, 26(8): 1341-1365, 2008.

[69] Ahmed W. K. M., P. J. McLane. "Random coding error exponents for flat fading channels with realistic channel estimation", IEEE Journal on Selected Areas in Communications, 18(3): 369-379, 2000.

[70] Ekpenyong A. E. and Y. F. Huang. "Feedback Constraints for Adaptive Transmission”, IEEE Signal Processing Magazine, 24(3): 69-78, 2007.

[71] Eriksson T. and T. Ottosson. "Compression of Feedback for Adaptive Transmission and Scheduling", Proceedings of the IEEE, 95(12): 23142321, 2007.

[72] Ekpenyong A. E. and H. Yih-Fang. "Feedback-detection strategies for adap- tive modulation systems", IEEE Transactions on Communications, 54(10): 1735- 1740, 2006.

[73] Kuhn M., Ettefagh A., Hammerstrom I., Wittneben A. "Two-way Communication for IEEE 802.11n WLANs Using Decode and Forward Relays", Fortieth Asilomar Conference on Signals, Systems and Computers, 2006.

[74] Cartwright, H. M. "The genetic algorithm in science, Pesticide Science", 45: 171C178. doi: 10.1002/ps.2780450212, 1995.

[75] Wan Z, Xiong N, Ghani N, Vasilakos AV, Zhou L (2012) Adaptive unequal protection for wireless video transmission over IEEE 802.11e networks. Multimedia Tools Appl. doi:10.1007/s11042-013-1378-z.

[76] Wei W, Zakhor A (2009) Interference aware multipath selection for video streaming in wireless ad hoc networks. IEEE Trans Circ Syst Video Technol 19(2):165-178. 